\title{
SELECTUS SEMINUM ANNO 1926 COLLECTORUM QUAE HORTUS BOTANICUS POSNANIENSIS MUTUO COMMUTANDA OFFERT.
}

Ashillea filipendulina Lam.

- millefolium L.

- ptarmica L.

- salicifolia Bess.

Aconitum Napellus L.

Acroclinium roseum Hook.

Actaea spicata L.

Adenophora Potanini Fisch.

Adenostyles alliariae Kern.

Adonis aestivalis L.

- autumnalis L.

Aegopodium podagraria L.

Aethusa cynapium L.

Agrimonia eupatoria L.

- odorata Mill.

Agrostemma githago L.

Agrostis alba L.

- spica venti $L$.

Aira caespitosa L.

Ajuga genevensis L.

Alchemilla silvestris Schm,

Alectorolophus major Rchb.

Alisma plantago L.

Alliaria officinalis Andrz.

Allium fistulosum L.

- montanum Schm.

- sativum L. (bulb.)

- scorodoprasum L. (bulb.)

- vineale L. (bulb.)

Alnus cordata C. Koch.

- incana Moench. f. aurea

Althaea officinalis L.

- rosea Cav.

- - - v. nigra
Alyssum calycinum L.

- saxatile L. compactum

Amarantus albus L.

- ascendens Loiss.

- caudatus L.

- - L. v. albiflorus

- paniculatus L.

- pan. L. X retroflexus L.

- retroflexus $\mathrm{L}$.

Ammobium alatum R. Br.

Anagalis arvensis L.

- coerulea Schreb.

Anchusa officinalis L.

Androsace elongata L.

- septentrionalis $\mathrm{L}$.

Anemone silvestris L.

Anethum graveolens L.

Angelica silvestris L.

Antennaria dioica Gärt.

Anthemis cotula L.

- nobilis L.

- ruthenica M. B.

Anthericum liliago L.

- ramosum L.

Anthoxanthum odoratum L. Anthriscus cerefolium Hoffm. - silvester Hofím.

Antirrhinum orontium L.

Aquilegia vulgaris $\mathrm{L}$.

- L L. v. rosea

Arabis albida Stev.

- arenosa Scop.

- hirsuta Scop.

Archangelica officinalis Hoffm. 
Arctium lappa L

- minus Bernh.

- tomentosum Mill.

Arenaria serpyllifolia L.

Armeria vulgaris Willd.

Arnica montana L.

Arrhenatherum elatius $M$. et

Koch

Artemisia absinthium L.

- campestris L.

- vulgaris $\mathrm{L}$.

Asclepias incarnata L.

- Cornuti Decn.

Asparagus officinalis $\mathrm{L}$.

Asperugo procumbens L.

Asperula tinctoria L.

Aster alpinus L.

- tripolium L.

Astragalus cicer L.

- glycyphyllos L

Astrantia major L.

Atriplex hortense L.

- patulum L.

Avena sterilis L.

Ballota nigra L.

Barbarea vulgaris $\mathrm{R} . \mathrm{Br}$.

Berberis aquifolium Pursh.

Berterea incana DC.

Berula angustifolia Koch.

Bidens cernuus L.

- leucanthus Willd.

- tripartitus L.

Blumenbachia Hieronymi Urb.

Borrago officinalis $\mathrm{L}$.

Brassica campestris L.

- juncea Hook. J. et Thoms.

- nigra L.

Briza maxima L.

- media L.

Bromus mollis $\mathrm{L}$.

- secalinus L.

- tectorum L.

Brunella grandiflora Jacq.

- vulgaris L.

Bryonia alba $\mathrm{L}$.

- dioica Jacq.

Bunias orientalis $\mathrm{L}$.

Butomus umbellatus L.
Calendula arvensis L.

- officinalis L.

Campanula carpathica Jacq.

- cervicaria L.

- glomerata L. acaulis

- - L. superba

- medium L.

- persicifolia L. v. alba

- sibirica L.

- trachelium L.

Cannabis sativa $\mathrm{L}$.

Capsella bursa pastoris Mnch.

Caragana arborescens Lam.

Capsicum annuum L.

Carduus acanthoides $\mathrm{L}$.

Carex hirta L.

- silvatica Huds.

Carlina acaulis L. v. caulescens

- vulgaris $\mathrm{L}$.

Carum Carvi L.

Carthamus tinctorius $\mathrm{L}$.

Celastrus orbiculatus Thbg.

Centaurea austriaca Willd.

- cyanus L.

- jacea L.

- rhenana Bor.

- - - v. alba

- ruthenica Lam.

- scabiosa L.

- serotina Bor.

- sulphurea Bor.

Cephalaria tatarica Schrad.

- uralensis R. et Sch.

Cerastium arvense L.

- semidecandrum L.

- tomentosum L.

Cerinthe major $\mathrm{L}$.

- minor L.

Chaerophyllum aromaticum $\mathrm{L}$.

- temulum L.

Chelidonium majus L.

_ _ _ v. laciniatum Mill.

Chenopodium album $\mathrm{L}$.

- ambrosioides L.

- Bonus Henricus L.

- polyspermum L.

Chondrilla juncea $\mathrm{L}$.

Chrysanthemum leucanthemum

- rotundifolium W. K. [L.

- segetum L. 
Cicer arietinum $\mathrm{L}$.

Cichorium intybus $\mathrm{L}$.

Cicuta virosa $\mathrm{L}$.

Cirsium arvense Scop.

- lanceolatum Scop.

- oleraceum Scop.

Citrullus colocynthis Schrad.

Claytonia perfoliata Don.

Clematis alpina Mill.

Cnicus benedictus L.

Cochlearia officinalis L.

Coix lacryma Jobi L.

Collomia grandiflora Dougl.

- linearis Nutt.

Commelina coelestis Willd.

Conium maculatum $\mathrm{L}$.

Convolvulus arvensis $\mathrm{L}$.

- elongatus Willd.

- tricolor L.

Coreopsis tinctoria Nutt.

- - - v. prolifica Rac.

Coriandrum sativum $\mathrm{L}$.

Corispermum hyssopifolium L.

Coronilla varia $\mathrm{L}$.

Cortusa Matthioli L.

Corydalis sempervirens Pers.

- vesicaria Pers.

Cosmea hybrida hort.

Crambe maritima L.

Crataegus nigra $W$. et $K$.

- pinnatifida Bge.

Crepis biennis L.

- tectorum L.

Cucubalus baccifer L.

Cuscuta epithymum L.

- europaea L.

- lupuliformis Krock.

Cynoglossum officinale L.

Cynosurus cristatus L.

Cytisus nigricans L.

Dactylis glomerata L.

Datura stramonium L.

- Tatula L.

Dahlia variabilis Desf.

Daucus carota L.

Delphinium consolida L.

- elatum L.

- oxysepalum Pax et Borb.
Dianthus Armeria L.

- barbatus L.

- caesius Sm.

- carthusianorum L.

- Caryophyllus L.

- deltoides L.

- plumarius L.

- Seguieri Vill.

- superbus L.

Dictamnus albus- $\mathrm{L}$.

Digitalis ambigua Murr.

- lanáta Ehrh.

- lutea L.

- purpurea L.

Dimorphotheca pluvialis Mnch.

Dipsacus laciniatus L. .

- pilosus L.

- silvester Huds.

- - - v. torsus.

Doronicum austriacum Jacq.

Dracocephalum Ruyschiana L.

Dryas octopetala L.

Echinops sphaerocephalus L.

Echinospermum lappula Lehm.

Echium vulgare L.

Elsholtzia Patrini Gcke.

Elymus arenarius L.

Epilobium angustifolium L.

- parviflorum Schreb.

Erigeron acer L.

- canadense L.

Erodium cicutarium L'Her.

Eryngium planum L.

Erysimum cheiranthoides L.

Eschscholzia californica Cham.

Eupatorium agerathoides $\mathrm{L}$.

- cannabinum L.

Euphorbia esula L.

- helioscopia L.

- lathyris L.

- palustris L.

- peplus L.

Euphrasia Rostkoviana Hayne Evonymus nana $M$. B.

Fagopyrum esculentum Mnch. - tataricum Gärt.

Falcaria vulgaris Bernh. 
Festuca amethystina Host.

- glauca Schrad.

- ovina L.

- pratensis Huds.

Filipendula hexapetala Gilib.

- vlmaria Max.

Foeniculum vulgare Mill.

Fumaria officinalis L.

- Vaillantii Lois.

Gaillardia aristata Pursh.

Galega officinalis L.

Galeopsis bifida Bngh.

- ladanum L.

- ochroleuca Lmk.

- pubescens Bess.

- tetrahit L.

Galinsoga parviflora Cav.

Galium aparine L.

- boreale L.

- mollugo L.

- Schultesii Vest.

- silvaticum L.

Genista tinctoria L.

Gentiana pneumonanthe L.

- uliginosa Willd.

Geranium pratense L.

- pusillum Burm.

- ruthenicum Uecht.

- sanginineum L.

Geum rivale L.

- rivale $X$ urbanum L.

- urbanum L.

Gilia tricolor Bentl.

Gladiolus imbricatus L.

Glaucium flavum Crantz.

Glyceria fluitans $\mathrm{R}$. Br.

Gnaphalium silvaticum L.

Gratiola officinalis L.

Gypsophila acutifolia Fisch.

- paniculata L.

Harpalium rigidum

Hedysarum obscurum L.

Helianthus annuus L.

- tuberosus L. (tub.)

Helianthemum grandiflorum

- obscurum Pers.

Lam, et DC.

Heliotropium europaeum L.
Helichrysum arenarium DC.

Heracleum sphondylium L.

Herniaria glabra L.

Hesperis matronalis L.

- tristis I.

Hieracium aurantiacum L.

- bupleuroides Gmel.

- lanatum W. et K.

- pilosella L.

- pratense Tausch.

- silvestre Tausch.

Holcus lanatus L.

- mollis L.

Holosteum umbellatum L.

Hyoscyamus albus L.

- niger $\mathrm{L}$.

Hypericum acutum Mnch.

-- perforatum L.

- quadrangulum L.

Hypochoeris glabra L.

- radicata L.

Hyssopus officinalis L.

Iberis amara $\mathrm{L}$.

Impatiens balsamina $\mathrm{L}$.

- glanduligera Royle

- parviflora DC.

Inula britannica $L$.

- Helenium L.

Iris pseudacorus L.

- sibirica L.

Ipomea purpurea Lam,

- rubro-coerulea Hook.

Isatis tinctoria $\mathrm{L}$.

Jasione montana L.

Juncus conglomeratus L.

- glaucus Ehrh.

- lamprocarpus Ehrh.

Knautia arvensis Coult.

Koeleria glauca DC.

Lactuca perennis L.

- saligna L.

- scariola L.

Lamium album L.

- amplexicaule L.

Lapsana communis L.

Lathyrus aphaca L.

- maritimus Big. 
Lathyrus ochrus DC.

- pratensis L.

- silvester L.

- vernus Bernh.

Lavandula vera DC.

Lavatera thuryngiaca L.

Lens esculenta Minch.

Leontodon hastilis L.

Leontopodiun alpinum Cass.

Lepidium draba L.

- ruderale $\mathrm{L}$.

- sativum L.

Levisticum officinale $\mathrm{L}$.

Libanotis montana Crantz.

Ligustrum Ibota S. et Z.

- Regelianum Koehne

- vulgare L.

Lilium candidum L.

- martagon L.

Linaria elatine Mill.

- vulgaris Mill.

Linum austriacum L.

- catharticum I.

-- perenne L.

- usitatissimum L.

Lithospermum arvense L.

- officinale $\mathrm{L}$.

Loasa tricolor Lindl.

- vulcanica André

Lunaria annua $\mathrm{L}$.

Lupinus albus L.

- angustifolius L.

- luteus L.

- perennis L.

- succulentus Doug1.

- varius L.

Luzula maxima DC.

Lychnis flos cuculi L.

- - Jovis Desr.

Lycopsis arvensis L.

Lysimachia punctata L.

- vulgaris L.

Lythrum hyssopifolia L.

- salicaria L.

Madia sativa Mol.

Malva alcea L.

- crispa L.

- moschata L.

- neglecta Wallr.
Malva rotundifolia $\mathrm{L}$. silvestris L.

Malope trifida Cav.

Marrubium vulgare $L$.

Matricaria chamomilla L.

- discoidea DC.

- inodora L.

Medicago falcata $\mathrm{L}$.

- falc. $X$ sativa $L$.

- lupulina L.

- sativa L.

Melandryum album Gke.

- noctiflorum Fries.

- rubrum Gke.

- Zawadzkii A. Br.

Melica nutans L.

Melilotus albus Desr.

- officinalis Med.

Melissa officinalis L.

Mentisa crispa L.

- pulegium L.

Mercurialis annua L.

Mimulus cardinalis Dougl

- hybridus hort. grandfl.

Mirabilis Jalapa L.

- longiflora L.

Monarda fistulosa L.

Montbretia crocosmiaeflora

Lem. (bulb.)

Myagrum perfoliatum L.

Myosotis alpestris Schm.

- arenaria Schrad.

- hispida Schldi.

- intermedia Link.

Myosurus minimus L.

Nasturtium officinale $\mathrm{R}$. Br.

- silvestre R. Br.

Nepeta cataria L.

Nicandra physaloides Gärtn.

Nicotiana paniculata L.

- glutinosa L.

- rustica L.

- Sanderae hort.

- tabacum L.

- silvestris Sp. et C.

Nigella arvensis L.

- damascena L.

- sativa L.

Nonnea pulla DC. 
Oenothera biennis L.

- odorata Jacq.

Omphalodes linifolia Mnch.

Onobrychis montana DC.

- viciaefolia Scop.

Ononis spinosa L.

Onopordon acanthium L.

Orchis latifolia L.

Origanum vulgare L.

Ornithopus sativus Brot.

Oxalis corniculata L.

- - L. v. atropurpurea

Panicum crus galli L.

- lineare Krock.

- miliaceum L.

- sanguinale L.

Papaver argemone L.

- dubium L.

- orientale L.

- rhoeas L.

- somniferum L.

- - L. monstrosum

Parietaria officinalis $\mathrm{L}$.

Parnassia palustris L.

Pastinaca sativa L.

Pentstemon alpinus

- barbatus Nutt.

- diffusus Dougl.

Peucedanum Oreoselinum

Phaseolus multiflorus Lam.

Phleum Bochmeri Wib.

Phytolacca decandra L.

Pimpinella magna $L$.

- saxifraga $L$.

Pisum arvense $\mathrm{L}$.

- sativum L.

Plantago lanceclata L.

- major L.

- - L. monstrosa

_- L. rubrifolia

- maritima L.

- media L.

- ramosa Aschers.

Poa nemoralis L.

- pratensis L.

Polemonium coeruleum L.

Polycnemum arvense L.

Polycarpum tetraphyllum L.
Polygala comosa Schkuhr.

Polygonum aviculare L.

- bistorta L.

- convolvulus L.

- hydropiper L.

- nodosum Pers.

- persicaria L.

Portulaca oleracea L.

Potentilla argentea L.

- recta L.

- rupestris $\mathrm{Cr}$.

Primula cortusoides L.

- officinalis Jacq.

- veris $L$.

Pulicaria vulgaris Gärt.

Pulsatilla patens Mill.

- pratensis Mill.

Pyrethrum cinerariaefolium Vis.

- roseum M. B.

Ranunculus acer L.

- aconitifolius L.

- auricomus L.

- sceleratus L.

- Steveni Andrz.

Raphanus sativus $L$.

Rapistrum perenne Berg.

Reseda luteola L.

- odorata L.

Rhodante Manglesii Ldl.

Rheum undulatum L.

Ricinus communis L.

Rubia tinctorum L.

Rudbeckia laciniata L.

Rumex acetosa L.

- acetosella L.

- crispus L.

- hydrolapathum Huds.

- patientia L.

- sanguineus L.

-. scutatus L.

Ruta graveolens L.

Salsola Kali L.

Salvia Hormium Batt.

- Batt. v. rubra

- glutinosa L.

- officinalis L.

- pratensis L.

- sclarea L. 
Salvia silvestris $\mathrm{L}$.

_- verticillata $\mathrm{L}$.

Sanguisorba minor Scop.

- officinalis L.

Saponaria officinalis L.

Satureja acinos Briq.

- clinopodium Briq.

- hortensis L.

Saxifraga aizoon Jacq.

- hieraciifolia W. K.

Scabiosa columbaria L.

- lucida Vill.

- ochroleuca L.

Scandix macrorhynchus

- pecten Veneris L.

Scirpus lacustris L.

Scleranthus annuus L.

- perennis L.

Scorzonera hispanica L.

- humilis L.

- purpurea L.

Scrophularia alata Gilib.

- nodosa $\mathrm{L}$.

- Scopolii Hoppe

Sedum acre L.

- carpathicum Reuss.

- reflexum L.

- spurium M. B.

Senecio paludosus L.

- subalpinus Koch.

- vernalis W. K.

- vulgaris $\mathrm{L}$.

Serratula tinctoria L.

Setaria glauca P. B.

- viridis $P$. B.

Sherardia arvensis $\mathrm{L}$.

Silene conica L.

- longiflora Ehrh.

- muscipula L.

- nutans L.

- otites Sm.

- tatarica Pers.

- venosa Aschers.

Silphium perfoliatum L.

Silybum Marianum Gärt.

Sinapis alba $\mathrm{L}$.

- arvensis L.

Sisymbrium officinale Scop.

- sinapistrum $\mathrm{Cr}$.
Sisymbrium Sophia L.

Sium latifolium L.

Solanum dulcamara L.

- luteum Mill.

- nigrum L.

Soldanella hungarica Simk

Solidago canadensis L.

- virga aurea L.

Sonchus arvensis L.

- asper Hill.

- oleraceus L.

Sorghum saccharatum Mnch.

- vulgare Pers.

Sparganium ramosum Court.

Specularia speculum A. DC.

Spergula arvensis L.

- vernalis Willd.

Spinacia oleracea L.

Stachys lanatus Jacq.

- officinalis Trev.

- rectus $\mathrm{L}$.

- palustris L.

Statice nana compacta

- Suworowii Reg.

Stellaria nemorum L.

Stenophragma Thalianum Cel,

Stupa capillata L.

- pennata L.

Succissa pratensis Mnch.

Sweertia alpestris Bmg.

Synuphytum officinale L.

Tagetes patula L.

- signata Bartl.

Tanacetum vulgare $\mathrm{L}$.

Telekia speciosa Bmg.

Teucrium chamaedrys L.

- scordium L.

- scorodonia L.

Thalictrum angustifolium L.

- aquilegifolium L.

- flavum L.

- minus L.

Thlaspi arvense L.

Thymus serpyllum Borb.

Tofieldia calyculata Wahlbg.

Torilis anthriscus Gmel.

Tragopogon major Jacq.

- pratensis L.

Trifolium alpestre L. 
Trifolium arvense $\mathrm{L}$.

- aureum Poll.

- badium L.

- lupinaster L.

- pratense L.

- repens L.

- rubens $\mathrm{L}$.

Triglochin maritimum L.

- palustre L.

Trigonella foenum graecum L

Triticum polonicum L.

- Spelta L.

- turgidum L.

- L. pseudocervinum

Tropaeolum majus $\mathrm{L}$.

Tunica prolifera Scop.

- saxifraga Scop.

Turritis glabra L.

Typha angustifolia L.

- latifolia L.

Urtica cannabina L.

- dioica L.

- pilulifera L.

Valeriana officinalis $\mathrm{L}$.

- sambucifolia Mik.

Valerianella coronata DC.

Verbascum lychnitis L.

- nigrum $L$.

- phlomoides L.

- phoeniceum L.

- thapsiforme Schrad.

Verbena officinalis L.

Posnaniae, Januarii 1927.

Prof. Dr. A. Wodziczko

horti botan. director.

J.W. Szulczewski

adjunctus.
Veronica arvensis L.

- beccabunga $\mathrm{L}$.

- chamaedrys L.

- longifolia L.

- officinalis L.

- saxatilis Scop.

- serpyllifolia L.

- teucrium L.

- triphyllos L.

Vicia angustifolia L.

- cassubica L.

- cracca L.

- Faba L.

- hirsuta Koch.

Vicia pisiformis L.

- sativa L.

- sepium L.

- tetrasperma Mnch.

- villosa Rth.

Vincetoxicum nigrum Mnch.

- officinale Mnch.

Viola hirta L.

- Riviniana Rchb.

- tricolor L.

Viscaria vulgaris Röhl.

Weingärtneria canescens Bernh.

Xanthium italicum Mor.

- spinosum $\mathrm{L}$.

Xeranthemum annuum L.

Zea Mays L.

Zinnia Haageana Regel
Wt. Marciniec

horti urban. director.

Wt. Drzewiecki

horti botan. inspector. 


\section{Hortus Botanicus Posnaniensis}

Poznań

Dąbrowskiego 165.

(Polonia)

\section{o \\ SELECTUS SEMINUM ANNO 1927 COLLECTORUM QUAE HORTUS BOTANICUS POSNANIENSIS MUTUO COMMUTANDA OFFERT. \\ DESIDERATA ANTE DIEM 15. II. 1928 NOBIS COMMUNICANDA SUNT.}

Alismataceae.

Alisma plantago L.

Sagittaria sagittifolia $\mathrm{L}$.

Amarantaceae.

Amarantus ascendens Loiss.

- caudatus L.

- L L. v. albiflorus

- paniculatus L.

- pan. L. X retroflexus L.

- retroflexus L.

Celosia cristata L.

Apocynaceae.

Vinca major L.

\section{Araceae.}

Acorus Calamus L.

Calla palustris $\mathrm{L}$.

Pinellia tuberifera Schott

\section{Asclepiadaceae.}

Asclepias Cornuti Decn.

- incarnata L.

Vincetoxicum nigrum Moench

- officinale Moench

\section{Balsaminaceae.}

Impatiens balsamina L.

- glanduligera Royle

- parviflora DC.

\section{Berberidaceae.}

Berberis vulgaris $L$.
Betulaceae.

Alnus glutinosa Gäert.

- cordata C. Koch

- incana Moench

- Moench. aurea hort.

Betula pubescens Ehrh.

- pub, Ehrh. X verrucosa

- verrucosa Ehrh. Ehrh.

\section{Borraginaceae.}

Anchusa officinalis L. Asperugo procumbens L. Borrago officinalis L. Cerinthe major $\mathrm{L}$.

- minor $\mathrm{L}$.

Cynoglossum officinale L. Echium vulgare L.

Heliotropium europaeum L.

- peruvianum L. hybr.

Lappula Myosotis Moench Lithospermum arvense $\mathrm{L}$. - officinale L.

Lycopsis arvensis $\mathrm{L}$.

Myosotis alpestris Schm.

- arenaria Schrad.

- hispida Schldl. intermedia Link.

Omphalodes linifolia Moench. Symphytum officinale L.

\section{Butomaceae.}

Butomus umbellatus L. 
Campanulaceae.

Adenophora Potanini Fisch. Campanula bononiensis L.

- carpathica Jacq.

- cervicaria L.

- glomerata L.

- L L acaulis

- L L. superba

- medium L.

- persicifolia L. alba

- pyramidalis L.

- rapunculoides $\mathrm{L}$.

- rotundifolia L.

- sibirica L.

- trachelium L.

Jasione montana L.

Phyteuma orbiculare L.

Specularia Speculum

\section{Caprifoliaceae.}

Veneris

A. DC.

Lonicera sempervirens L. cocc.

Symphoricarpus orbiculatus sup. Moench.

- racemosus Michx.

Viburnum Lantana L.

\section{Caryophyllaceae.}

Agrostemma githago L.

Arenaria serpyllifolia L.

Cerastium semidecandrum L

Cucubalus baccifer L.

Dianthus Armeria L.

- barbatus L.

- caesius Sm.

- carthusianorum L.

- Caryophyllus L.

- deltoides L.

- plumarius L.

- praecox W. K.

- Seguieri Vill.

- squarrosus Bieb.

- superbus L.

Gypsophila acutifolia Fisch. - paniculata L.

Heliosperma quadrifida Rchb.

Herniaria glabra L.

Holosteum umbellatum L.

Lychnis flos cuculi L.

- Jovis Desr.
Melandryum album Gke.

- noctiflorum Fries.

- rubrum Gke.

- Zawadzkii A. Br.

Saponaria ocymoides L.

- officinalis $\mathrm{L}$.

Scleranthus annuus L.

- perennis L.

Silene acaulis $\mathrm{L}$.

- conica L.

- muscipula L.

- nutans L.

- otites $\mathrm{Sm}$.

- schafta Gmel.

- tatarica Pers.

- viridiflora L.

- venosa Aschers.

Spergula arvensis L.

- vernalis Willd.

Stellaria graminea L.

- nemorum L.

Tunica prolifera Scop.

- saxifraga Scop.

Viscaria vulgaris Röhl.

\section{Celastraceae.}

Celastrus orbiculatus Thbg. Evonymus europaea L. - nana M. B.

\section{Chenopodiaceae.}

Atriplex hortense L.

- patulum L.

Chenopodium album L.

- ambrosioides L.

- Bonus Henricus L.

- hybridum L.

- polyspermum L.

Corispermum hyssopifolium L. Salicornia herbacea L.

Salsola Kali L.

Spinacia oleracea L.

\section{Cistaceae.}

Helianthemum grandiflorum

- obscurum Pers.

$$
\text { Lam. et DC. }
$$

- poliifolium DC. 


\section{Compositae.}

Achillea filipendulina Lam.

- millefolium $\mathrm{L}$.

- L L. v. rubra

- ptarmica L.

- salicifolia Bess.

Antennaria dioica Gaert.

Anthemis nobilis L.

- ruthenica M. B.

Arctium lappa L.

- minus Bernh.

- nemorosum Lej.

- tomentosum Mill.

Arnica montana L

Artemisia absinthium L.

- campestris L.

- vulgaris $\mathrm{L}$.

Aster alpinus L.

- tripolium L.

Bellis perennis $\mathrm{L}$

Bidens cernuus L.

- tripartitus L.

Calendula arvensis $\mathrm{L}$. officinalis $\mathrm{L}$.

Carduus acanthoides $\mathrm{L}$.

- cernuus Benth.

- defloratus auct.

Carlina acaulis L. v. caulescens

- vulgaris L.

Carthamus tinctorius I.

Centaurea austriaca Willd.

- cyanus L.

- jacea L.

- rhenana Bor.

- Bor. v. alba

- ruthenica Lam.

- scabiosa Bor.

- sulphurea Bor.

Chondrilla juncea L.

Chrysanthemum leucanthemum L. segetum $\mathrm{L}$.

Cichorium endivia L.

- intybus L.

Cirsium acaule Al1.

- arvense Scop.

- erisithales Scop.

- lanceolatum Scop.

- oleraceum Scop.

- palustre Scor.

Cnicus benedictus $L$.
Coreopsis tinctoria Nutt.

- - Nutt, v. prolifica Rac.

- grandiflora Nutt.

Cosmos bipinnatus Cav.

Crepis biennis L.

- Jacquini Tausch.

- tectorum L.

Dahlia variabilis Desf.

Dimorphotheca pluvialis Moench

Doronicum austriacum Jacq.

Echinops sphaerocephalus L.

Erigeron acer L.

- canadense L.

Eupatorium agerathoides L.

- cannabinum L.

Gaillardia aristata Pursh.

Galinsoga parviflora Cav.

Helianthus annuus L.

tubarosus L. (tub.)

Helichrysum arenarium DC

Hieracium aurantiacum L.

- bupleuroides Gmel.

- lanatum W. et K.

- pilosella L.

- sabaudum L.

- umbellatum L.

- L. f. maritima

- vulgatum Fries.

Hypochoeris glabra L.

- maculata L.

- radicata $L$.

Inula britannica $\mathrm{L}$.

- Helenium L.

Lactuca muralis Less.

- perennis L.

- saligna L.

- scariola L.

Lapsana communis $\mathrm{L}$.

Leontopodium alpinum Cass.

Leontodon autumnalis L.

- hispidus L.

Madia sativa Mol.

Matricaria chamomilla L.

- discoidea DC.

Monarda fistulosa L.

Onopordon acanthium L.

Picris hieracioides $L$.

Prenanthes ourpurea L

Pulicaria vulgaris Gaert.

Pyrethrum cinerariaefolium Vis. 
Rhodante Manglesii Ldl.

Senecio Fuchsii Gmel.

- Jacobaea L.

- paludosus L.

- subalpinus Koch

- vernalis W. K.

- vulgaris L.

Scorzonera hispanica L.

- purpurea L.

Serratula tictoria L.

Siegesbeckia orientalis L.

Silphium perfoliatum L.

Silybum marianum Gaert.

Solidago virga aurea $\mathrm{L}$.

Sonchus arvensis L.

- asper Hill.

- oleraceus L.

Tagetes signata Bartl.

Tanacetum vulgare $L$.

Taraxacum lepidophyllum Dahlst. - officinale Web.

Telekia speciosa Baumg.

Tragopogon dubius Scop.

- porrifolius L.

pratensis $\mathrm{L}$.

Xanthium italicum Mor.

- spinosum L.

Xeranthemum annuum L.

\section{Commelinaceae.}

Commelina coelestis Willd

Tradescantia virginica $L$.

\section{Convolvulaceae.}

Calystegia sepium K. Br.

Convolvulus arvensis $\mathrm{L}$.

- elongatus Willd.

- tricolor L.

Ipomea purpurea Lam. - rubro - coerulea Hook,

Cuscuta epithymum L.

- europaea L.

- lupuliformis Krock.

\section{Cornaceae.}

Cornus alba L.

\section{Crassulaceae.}

Sedum acre L.

- carpathicum Reuss.
Sedum maximum Sut.

- reflexum L.

- spurium M. B.

Sempervivum montanum $\mathrm{L}$.

- soboliferum L.

\section{Cruciferae.}

Alliaria officinalis, Andrz.

Alyssum calycinum L.

- montanum L.

- saxatile L compactum.

Arabis alpina L.

- arenosa Scop.

- bellidifolia Jacq.

- Halleri L.

- hirsuta Scop.

Barbaraea vulgaris $\mathrm{R}, \mathrm{Br}$.

- R. Br. fol. var.

Berteroa incana DC.

Brassica campestris L.

- juncea Hook. J. et Thoms.

- nigra Koch

- napus L. oleifera

- rapa $L$. oleifera

Bunias orientalis L.

Camelina microcarpa Andrz.

- sativa Crantz

Capsella Bursa pastoris Mnch.

Cochlearia officinalis $\mathrm{L}$.

Cheiranthus cheiri L

Erysimum cheiranthoides L.

Hesperis matronalis L.

- tristis L.

Iberis amara $\mathrm{L}$.

Isatis tinctoria L.

Lepidium draba L.

- ruderale $\mathrm{L}$.

- sativum L.

Lunaria annua $\mathrm{L}$.

Myagrum perfoliatum L.

Nasturtium silvestre $\mathrm{R}, \mathrm{Br}$.

Raphanus sativus $\mathrm{L}$

Rapistrum perenne Berg.

Sinapis alba L.

- arvensis L.

Sisymbrium ctficinale Scop.

- sinapistrum Crantz

- Sophia L.

Stenophragma Thalion'sm Cel.

Thlaspi arvense $\mathrm{L}$.

Turritis glabra $\mathrm{L}$. 


\section{Cucurbitaceae.}

Bryonia alba L. dioica Jacq.

Citrullus colocynthis Schrad.

\section{Cyperaceae.}

Carex acutiformis Ehrh.

- arenaria L.

- atrata L.

- contigua Hoppe

- Goodenoughii Gay.

- hirta L.

- leporina L.

- limosa L.

- pallescens L.

- paniculata L.

- pseudocyperus L.

- riparia Curt.

- secalina Wahlbe.

- sempervirens Vill.

- silvatica Huds.

- vesicaria $L$.

Cladium mariscus $\mathrm{R}$. $\mathrm{Br}$. Eriophorum polystachium L. Scirpus lacustris L.

- maritimus L. v. compactus

- silvaticus L.

Dipsacaceae.

Cephalaria tatarica Schrad. - uralensis R. et Sch.

Dipsacus pilosus L. - silvester Huds.

Knautia arvensis Coult.

Scabiosa columbaria L̃.

- lucida Vill.

- ochroleuca L.

Succisa pratensis Mnch

\section{Droseraceae.}

Drosera rotundifolia L.

\section{Ericaceae.}

Erica tetralix L.

\section{Euphorbiaceae.}

Euphorbia esula L.

- helioscopia L.

- Lathyris L.

- palustris L.

- peplus L.
Mercurialis annua L.

Ricinus communis L.

\section{Gentianaceae.}

Centaurium umbellatum Gilib.

Gentiana asclepiadea L.

- Clusii Perr, et Song.

- lutea L.

- lutescens Vel.

- pneumonanthe L.

Sweertia alpestris Bmg.

\section{Geraniaceae.}

Erodium cicutarium L'Her.

Geranium phaeum L.

- pratense L.

- pusillum Burm.

- Robertianum L.

- sanguineum L.

\section{Gramineae.}

Agrostis alba L.

- vulgaris With.

- spica venti $L$.

Aira caespitosa L.

Alopecurus pratensis L.

Anthoxantum odoratum L.

Arrhenatherum bulbosum Schldl. fol. var.

- elatius M. et Koch

Briza maxima L.

- media L.

Bromus inermis Leyss.

- mollis L.

- secalinus L.

- sterilis L.

- tectorum L.

Calamagrostis arundinacea $\mathrm{Rth}$.

- epigeios Rth.

Cynosurus cristatus L.

Dactylis glomerata L.

Elymus arenarius L.

Festuca amethystina L. v. Rit-

- glauca Schrad. schlii Hackel

- ovina L.

- pratensis Huds.

Glyceria aquatica Whlb.

- distans Whlb.

- fluitans R. Br.

Holcus mollis L. 
Hordeum murinum L.

Koeleria glauca DC.

- gracilis Pers.

Lolium temulentum L.

- perenne L.

Melica nutans L.

Milium effusum L.

Panicum crus galli L.

- lineare Krock.

- miliaceum L.

- sanguinale $\mathrm{L}$.

Phalaris arundinacea L. v. picta Phleum Boehmeri Wib.

- pratense L.

Poa nemoralis L.

- pratensis L.

Setaria glauca P. B.

Stupa capillata L.

- pennata L.

Triticum caninum L.

- polonicum L.

- repens L.

- spelta L.

- turgidum L.

- - L. v. pseudocervinum

Weingaertneria canescens Bernh.

\section{Guttiferae.}

Hypericum acutum Moench.

- quadrangulum L.

- montanum L.

\section{Hippocastanaceae.}

Aesculus hippocastanum L.

\section{Iridaceae.}

Gladiolus imbricatus L.

Iris pseudoacorus L.

- sibirica L.

Sisyrinchium angustifolium Mill.

Montbretia crocosmiaeflora

Lem. (bulb).

\section{Juglandaceae.}

Juglans cinerea $\mathrm{L}$.

- nigra L.

\section{Juncaginaceae.}

Scheuchzeria palustris L.

Triglochin marítimum $\mathrm{L}$

- palustre L.
Juncaceae.

Juncus bufonius L.

- conglomeratus L.

- compressus Jacq.

- effusus L.

- glaucus Ehrh.

\section{Labiatae.}

Ajuga genevensis L

Ballota nigra $L$

Brunella grandiflora Jacq. vulgaris $L$.

Calamintha acinos Clairv.

- clinopodium Benth.

Dracocephalum Ruyschian? L.

Elsholtzia Patrini Gcke.

Galeobdolon luteum Huds.

Galeopsis bifida Boenn.

Galeopsis ladanum L.

- ochroleuca Lmk.

- pubescens Bess.

Hyssopus officinalis L

Lamium album $\mathrm{L}$.

- amplexicaule L.

- maculatum L.

Lavandula vera $\mathrm{DC}$.

Leonurus cardiaca L.

Lycopus europaeus L.

Marrubium vulgare $\mathrm{L}$.

Mentha arvensis L.

- pulegium L.

- silvestris L.

Melissa officinalis L.

Monarda fistulosa L.

Nepetha cataria L.

Origanum majorana L

- vulgare L.

Phlomis alpina Pall.

Salvia argentea L.

- cleistogama De Barry et

- Hormium Batt. Pau!.

- Batt. v. rubra

- glutinosa L.

- officinalis L.

- pratensis L.

- sclarea L.

- silvestris L.

- verticillata L.

Satureja hortensis L. 
Scutellaria altissima L

- galericulata L.

Stachys lanatus Jacq.

- officinalis Trev.

- palustris L.

- rectus L.

- silvaticus L.

Teucrium chamaedrys L.

- scorodonia L.

Thymus serpyllum Borb.

\section{Liliaceae.}

Allium fistulosum L

- montanum Schm.

- schoenooprasum L.

- Scorodoprasım L. (bulb.)

- vineale L. (bulb.)

Anthericum liliago L

- ramosum L.

Asparagus officinalis $\mathrm{L}$.

Lilium martagon L.

Scilla bifolia L.

- sibirica Andrz.

Tofieldia calyculata Wahlbg.

\section{Linaceae.}

Linum austriacum L.

- perenne L.

- usitatissimum L.

\section{Lythraceae.}

Lythrum hyssopifolia L.

- salicaria L.

\section{Malvaceae.}

Althaea officinalis L.

- rosea Cav.

- Cav. fl. pleno

- Cav. v. nigra.

Lavatera thuryngiaca L.

Malva alcea $\mathrm{L}$.

- crispa L.

- moschata L.

- neglecta Wallr.

- rotundifolia L.

- silvestris L.

\section{Moraceae.}

Cannabis sativa $\mathrm{L}$.
Oenotheraceae.

Circaea lutetiana L.

Epilobium angustifolium L.

- collinum Gmel.

- hirsutum L.

- montanum L.

- parviflorum Schreb.

- roseum Schreb.

Oenothera biennis L.

- Lamarckiana Pers.

\section{Oleaceae.}

Ligustrum Ibota S. et Z .

- Regelianum hort.

- vulgare L.

Syringa vulgaris $\mathrm{L}$

\section{Orchidaceae.}

Cypripedium calceolus L.

Epipactis latifolia All.

- palustris $\mathrm{Cr}$.

- rubiginosa Gaud.

Orchis latifolius L.

\section{Oxalidaceae.}

Oxalis corniculata L. atropur-

- stricta L.

purea

\section{- Papaveraceae.}

Bocconia cordata Willd.

Chelidonium majus L.

- - L. laciniatum

Corydalis sempervirens Pers

- vesicaria Pers.

Eschscholzia californica Cham.

Fumaria officinalis L.

- Vaillantii Lois.

Glaucium corniculatum Curt.

- flavum Crantz

Papaver argemone L.

- dubium L.

- nudicaule L.

- orientale L.

- rhoeas L.

- - L. fl. pleno

- somniferum L.

- L L. fl. pleno

- L L. v. monstrosum 


\section{Papilionaceae.}

Amorpha canescens Nutt. Anthyllis alpestris Kit.

- Kerneri Sag.

Astragalus cicer L.

- glycyphyllos L.

Caragana arborescens Lam.

Colutea arborescens L.

Cytisus nigricans L.

- ruthenicus Fisch.

Galega officinalis L.

Genista tinctoria L.

Laburnum vulgare Gris.

Lathyrus aphaca L.

- maritimus Big.

- niger Bernh.

- odoratus L.

- paluster L.

- pratensis L.

- silvester L.

- vernus Bernh.

Lens esculenta Mnch.

Lupinus angustifolius L.

- luteus L.

- mutabilis Lindl.

- polyphyllus Lindl.

- succulentus Dougl.

$\rightarrow$ varius $\mathrm{L}$.

Medicago falcata L.

- fakc. X sativa L.

- lupulina L.

- sativa L.

Melilotus albus Desr.

- dentatus Pers.

- officinalis Med.

Onobrychis viciaefolia Scos.

Ononis hircina Jacq.

- spinosa L.

Ornithopus sativus Brot.

Phaseolus multiflorus L.

Pisum arvense $\mathrm{L}$. sativum $\mathrm{L}$.

Trifolium alpestre $\mathrm{L}$.

- arvense L.

- aureum Poll.

- hybridum L.

- lupinaster L.

- montanum L.

- pratense L.

- repens L.
Trifolium resupinatum $\mathrm{L}$.

- rubens L.

Vicia cassubica L.

- cracca L.

- Faba L.

- dumetorum L.

- hirsuta Koch

- pisiformis L.

- sativa L.

- sepium L.

- tetrasperma Mnch.

Phytolaccaceae.

Phytolacca decandra L.

Plantaginaceae.

Plantago lanceolata L

- maior $\mathrm{L}$.

- - L. monstrosa

- - L. rubrifolia

- maritima $\mathrm{L}$

- maxima Ait.

- media L.

- ramosa Aschers.

\section{Plumbaginaceae.}

Armeria vulgaris Willd.

Statice incana

- nana compacta

Polemoniaceae.

Collomia grandiflora Dougl.

- linearis Nutt.

Polemonium coeruleum L.

Polygalaceae.

Polygala comosa Schkuhr - vulgaris $\mathrm{L}$.

\section{Polygonaceae.}

Fagopyrum escrlentum Mnch.

- tataricum Gaert.

Polygonum aviculare $\mathrm{L}$.

- bistorta L.

- convolvulus L.

- hydropiper L.

- nodosum Pers.

- persicaria L.

- viviparum $\mathrm{L}$.

Rheum undulatum $\mathrm{L}$. 
Rumex acetosa L.

- acetosella L.

- conglomeratus Murr.

- crispus L.

- hydrolapathum Huds.

- patientia L.

- scutatus L.

- silvestris Wallr.

\section{Portulacaceae.}

Claytonia perfoliata Don. Portulaca oleracea L.

\section{Potamogetonaceae.}

Potamogeton alpinus Balb.

\section{Primulaceae.}

Anagalis arvensis L.

- coerulea Schreb.

Androsace elongata L. - septentrionalis L.

Lysimachia punctata L. - vulgaris L.

Primula carpathica Fuss.

- cortusoides L.

- officinalis Jacq.

\section{Ranunculaceae.}

Aconitum Napellus L.

Actaea spicata L.

Adonis aestivalis $\mathrm{L}$.

- autumnalis L.

- vernalis L.

Anemone silvestris L.

Aquilegia chrysantha

- Helenae hort.

- vulgaris $\mathrm{L}$.

- - L. v, rosea

Caltha palustris $\mathrm{L}$.

Clematis alpina Mill.

- integrifolia L.

- vitalba $\mathrm{L}$.

- viticella $L$.

Delphinium consolida L.

- elatum L.

- oxysepalum Pax et Borb.

Myosurus minimus L.

Nigella arvensis L.

- damascena L.

- sativa L.
Pulsatilla alba Rchb.

- nigricans Störck.

- patens Mill.

Ranunculus acer L.

- aconitifolius L.

- arvensis L.

- auricomus L.

- bulbosus L.

- Ficaria L. (bulb.)

- Flamula L.

- lanuginosus L.

- platanifolius L.

- polyanthemos L.

- repens L.

- sardous Cr.

- sceleratus L.

Thalictrum angustifolium $\mathrm{L}$.

- aquilegifolium L.

- flavum L.

- minus L.

- simplex L.

Trollius europaeus L.

\section{Resedaceae.}

Reseda luteola L.

- odorata L.

- phyteuma L.

\section{Rhamnaceae.}

Rhamnus cathartica L. - frangula L.

\section{Rosaceae.}

Agrimonia eupatoria L. - odorata Mill.

Alchemilla pubescens Lam.

- silvestris Schm.

Amelanchier ovalis Med.

Aruncus silvester Kost,

Cotoneaster horizontalis Decne

- acutifolia Turcz.

Dryas octopetala L.

Filipendula hexapetala Gilib.

- ulmaria Max.

Geum montanum L.

- rivale $\mathrm{L}$.

- riv. L. X urbanum L.

- urbanum L.

Holodiscus discolor Max 
Potentilla argentea L.

- aurea L.

- fruticosa L.

- procumbens Sibth.

- recta $\mathrm{L}$.

- reptans L.

- rupestris $\mathrm{Cr}$.

Physocarpus opulifolius Max. - opulif. Max f. aurea

Rhodotypus kerrioides $\mathrm{S}$, et $\mathrm{Z}$.

Rosa mollis Sm.

- tomentosa Sm.

- rubrifolia Vill.

- rugosa Thunb.

Rubus seebergensis Pfuhl

Sanguisorba minor Scop. - officinalis L.

\section{Rubiaceae.}

Asperula tinctoria L.

Galium anisophyllum Vill.

- aparine L.

- boreale L.

- mollugo L.

- Schulthesii Vest.

Rubia tinctorum L.

Sherardia arvensis L.

\section{Rutaceae.}

Dictamnus albus L.

Ruta graveolens L.

\section{Saxifragaceae.}

Parnassia palustris L.

Ribes sang ineum Pursh.

Saxifraga Aizoon Jacq.

- aizoides L.

- androsacea L.

- adscendens L.

- bryoides L.

- granulata L.

- hieraciifolia W. K.

\section{Scrophulariaceae.}

Alectorolophus major Rchb.

Antirrhinum majus L.

- orontium L.

Digitalis ambigua Murr.

- lanata Ehrh.

- lan. Ehrh. X lutea L.
Digitalis lutea L.

- purpurea L.

- L. peloria.

Gratiola officinalis L

Linaria elatine Mill.

- genistifolia Mill.

- vulgaris Mill.

Mimulus cardinalis Dougl.

- hybridus hort. grandiflorus

- - hort. duplex

- tigrinus

Pentstemon alpinus Torr.

- azureus

- barbatus Roth.

-- diffusus Dougl.

- Gordoni Hook. splendens

Scrophularia alata Gilib.

- nodosa L.

- Scopolii Hoppe

Veronica arvensis L.

- anagallis L.

- beccabunga I.

- chamaedrys L.

- longifolia L.

- officinalis L.

- orchidea $\mathrm{Cr}$.

- saxatilis Scop.

- serpyllifolia L.

- spicata L.

- teucrium L.

- triphyllos L.

Verbascum lychnitis L.

- nigrum L.

- phoeniceum L.

- phlomoides L.

- thapsus L.

- thapsiforme Schrad.

\section{Solanaceae.}

Atropa Belladonna L.

Capsicum annuum L.

Datura stramonium L.

- Tatula L.

Hyoscyamus albus L.

- niger L.

Nicandra physaloides Gärt.

Nicotiana paniculata L.

- petiolaris Schlecht.

- rustica L

- Sanderae hort.

- tabacum L. 
Physalis Alkekengi L.

Solanum dulcamara L.

- luteum Mill.

- nigrum L.

\section{Sparganiaceae.}

Sparganium ramosum Court.

- simplex Huds.

\section{Thymelaeaceae.}

Daphne mezereum L.

\section{Typhaceae.}

Typha angustifolia L. - latifolia L.

\section{Umbelliferae.}

Anethum graveolens L. Angelica silvestris L.

Anthriscus cerefolium Hoffm. - silvester Hoffm.

Archangelica officinalis Hoffm. Astrantia major L.

Carum Carvi L.

Chaerophyllum aromaticum $\mathrm{L}$. - temulum L.

Cicuta virosa $\mathrm{L}$.

Conium maculatum L.

Daucus Carota L.

Eryngium maritimum L. planum L.

Falcaria vulgaris Bernh.

Foeniculum vulgare Mill.

Heracleum sphondylium L.

Levisticum officinəle L.

Libanotis montana $\mathrm{Cr}$.

Meum Muttelina Gaert.

Oenanthe Phellandryum DC.
Pastinaca sativa L.

Petroselinum sativum L.

Peucedanum Oreoselinum Mnch.

Sanicula europaea L.

Scandix macrorhynchus C. A.

- pecten Veneris L. Mey.

Torilis anthriscus Gmel.

\section{Urticaceae.}

Parietaria officinalis $\mathrm{L}$.

Urtica cannabina L.

- dioica L.

- Dodartii L.

- pilulifera L.

\section{Valerianaceae.}

Valeriana officinalis $\mathrm{L}$.

- Phu L.

- sambucifolia Mik.

Valerianella olitoria Poll.

\section{Verbenaceae.}

Verbena hybrida hort. grdfl.

- officinalis L.

- venosa Gill et Hook.

\section{Violaceae.}

Viola arvensis Murr.

- canina Rchb.

- elatior Fr.

- hirta L.

- mirabilis L.

- odorata L.

- Riviniana Rchb.

- silvestris Rchb.

tricolor L.

- - L. f. maritima

Posnaniae, Januarii 1928.

Prof. Dr. A. Wodziczko horti botan. director.
Wt. Drzewiecki horti botan. inspector.
J. W. Szulczewski adjunctus. 


\section{La Direction des Jardins Communaux} de la Ville de Poznań

augmente et complète la collection des plantes exotiques de ses serres. Tous ceux, qui veulent en échanger ou vendre, sont priés de s'adresser

à la „Direction des Jardins de la Ville de Poznan (Pologne)".

On envoie sur demande une liste des plantes, qui peuvent être échangées.

Wt. Marciniec, directeur. 


\section{HORTUS BOTANICUS}

POSNANIENSIS

POZNAŃ-POLONIA

DĄBROWSKIEGO 165.

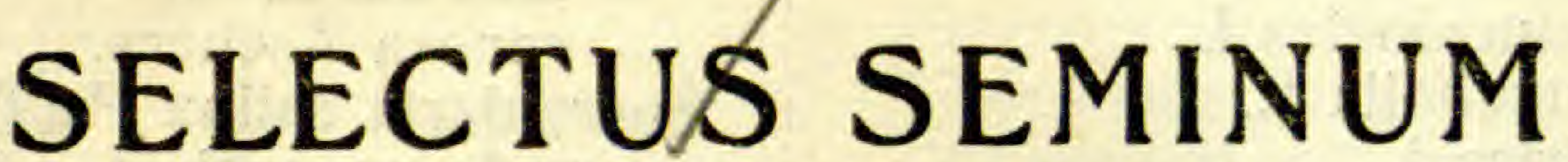

ANNO 1928 COLLECTORUM

\section{QUAE HORTUS BOTANICUS POSNANIENSIS MUTUO COMMUTANDA OFFERT.}

DESIDERATA ANTE DIEM 1. III. 29 NOBIS COMMUNICANDA SUNT.

Aceraceae.

Acer campestre L.

- Lobelii Ten.

- plantanoides L. var. Reitenbachii Nichols.

- tataricum L.

\section{Alismataceae.}

Sagittaria sagittifolia L.

\section{Amarantaceae.}

Amarantus ascendens Loiss.

- caudatus L.

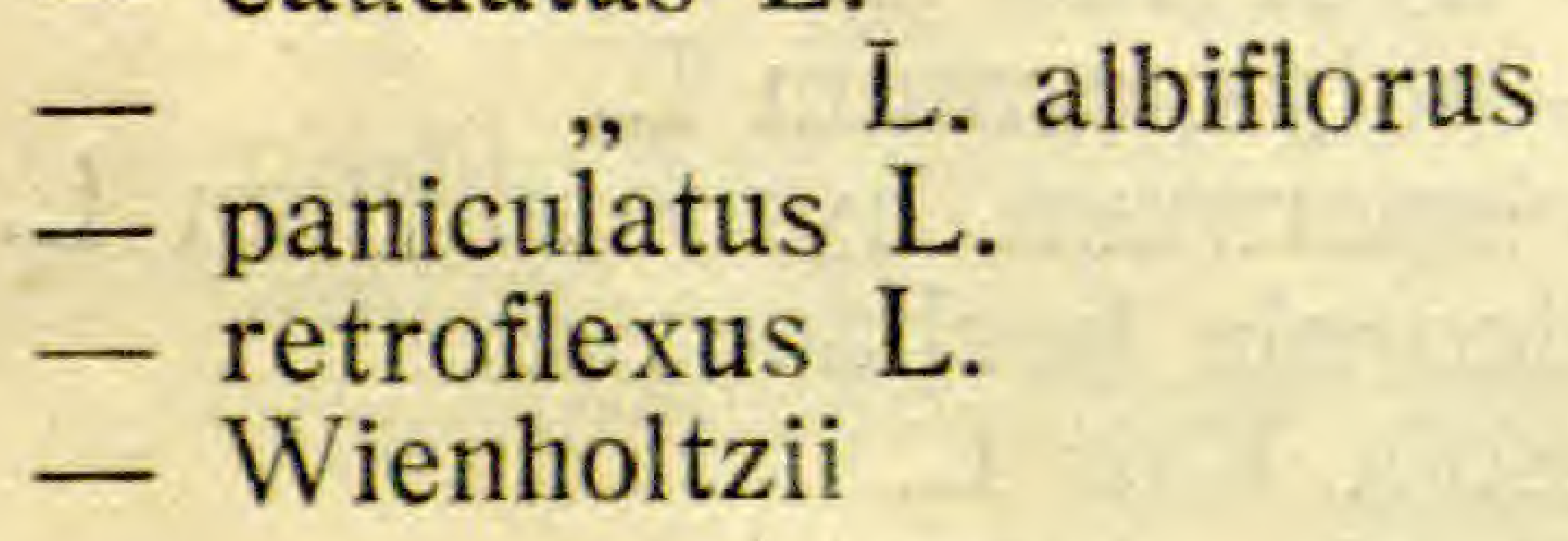

\section{Amaryllidaceae.}

Leucojum carpaticum Borb.

\section{Asclepiadaceae.}

Asclepias Cornuti Decn.

Vincetoxicum nigrum Moench

- officinale Moench

\section{Berberidaceae.}

Berberis aggregata C. S.

- Berberis Thunbergi DC.

- vulgaris L. f. atropurp.

\section{Betulaceae.}

Alnus cordata C. Koch

- glutinosa Gaert.
Alnus incana Moench Betula Ermanni Cham.

- humilis L.

- nana L.

- oycoviensis Bess.

- papyracea Ait.

- pubescens Ehrh.

- verrucosa Ehrh.

- pubesc. verrucosa

Carpinus betulus L.

Borraginaceae.

Anchusa officinalis L.

Asperugo procumbens L.

Borago officinalis $\mathrm{L}$.

Cerinthe minor L.

Cynoglossum officinale $\mathrm{L}$.

Echium vulgare $L$.

-,$\quad$ L. roseum

Heliotropium europaeum L.

Lappula Myosotis Moench

- deflexa Gicke.

Lithospermum arvense L.

- officinale L.

Lycopsis arvensis $\mathrm{L}$.

Myosotis alpestris Schm.

- hispida Schm.

- intermedia Link.

Nonnea pulla DC.

Symphytum officinale L.

\section{Campanulaceae.}

Adenophora Potanini Fisch. Campanula bononíenśis L.

FEB 16 1923, 
Campanula carpatica Jacq.

- cervicaria L.

- glomerata L.

- medium L.

- persicifolia L. alba

- pyramidalis L.

- rapunculoides L.

- rotundifolia L.

- sibirica L.

- trachelium L.

Jasione montana L.

Pytheuma orbiculare L.

Specularia Speculum Veneris

A. DC.

\section{Caprifoliaceae.}

Lonicera sempervirens L. cocc.

- xylosteum L.

\section{superba}

Symphoricarpus Heyeri Dipp.

- orbiculatus Moench

- racemosus Michx.

\section{Caryophyllaceae.}

Agrostemma githago L. Arenaria serpyllifolia L.

Cerastium arvense $L$.

- semidecandrum L.

Dianthus Armeria L.

- barbatus L.

- caesius Sm.

- carthusianorum L.

- deltoides L.

- plumarius L.

- praecox W. K.

- Seguieri Vill.

- serotinus W. K.

- superbus L.

Gypsophila acutifolia Fisch.

- fastigiata L.

- paniculata L.

- repens $L$.

Herniaria glabra L.

Holosteum umbellatum L.

Lychnis flos cuculi L.

Jovis Desr.

Melandryum album Gcke.

- noctiflorum Fries

- rubrum Gcke.

- Zawadzkii A. Br.
Saponaria ocymoides L.

- officinalis L.

Scleranthus perennis $\mathrm{L}$.

Silene conica L.

- otites Sm.

- tatarica Pers.

- venosa Aschers.

Spergularia salina Presl.

Spergula arvensis 'L.

- vernalis Willd.

Stellaria graminea $\mathrm{L}$.

- nemorum L.

Tunica prolifera Scop.

- axifraga Sco ?

Viscaria vulgaris Röhl.

\section{Celastraceae.}

Celastrus orbiculatus Thbg.

Evonymus alata C. Koch.

- europaea L.

\section{Chenopodiaceae.}

Atriplex hortense L.

- hastatum L. var. salinum

Chenopodium album L.

- ambrosioides L.

- bonus Henricus L.

- hybridus L.

- polyspermum L.

Corispermum hyssopifolium L.

Salicornia herbacea L.

Salsola Kali L.

Spinacia oleracea L.

\section{Cistaceą.}

Helianthemum grandiflorum

- obscurum Pers.

\section{Lam. et DC.}

\section{Compositae.}

Achillea distans W. K.

- millefolium L.

- millefolium L. var. rubra.

- pannonica Scheele

- ptarmica L.

- salicifolia Bess.

Acroclinium roseum Hook.

Antennaria dioica Gaert.

Anthemis nobilis L. 
Anthemis ruthenica M. B.

Arctium lappa L.

- minus Bernh.

- nemorosum Lej.

- tomentosum Mill.

Arctotis grandis Thbg.

Arnica montana L.

Aster alpinus L.

Bidens cernuus $\mathrm{L}$.

- tripartitus L.

Calendula arvensis L.

- officinalis L.

Carduus acanthoides L.

- cernuus Benth.

- crispus L.

- glaucus Bmg.

- Kerneri Simk.

Carlina acaulis L. var. caules.cens.

- vulgaris L.

Carthamus tinctorius $\mathrm{L}$. Centaurea austriaca Willd.

- cyanus L.

- jacea L.

- Kotschyana Heuff.

- oxylepis Hay.

- ruthenica Lam.

- scabiosa L.

- serotina Bor.

- sulphurea Bor.

Chondrilla juncea L.

Chrysanthemum leucanthe-

- rotundifolium W. K. mum $\mathrm{J}$.

- segetum L.

Cichorium intybus $\mathrm{L}$.

Cirsium acaule All.

- arvense Scop.

- erisithales Scop.

- lanceolatum Scop.

- palustre Scop.

Coreopsis tinctoria Nutt. v. pro-

- grandiflora Nutt. lifica Rac.

Cosmos bipinnatus Cav.

Crepis biennis $\mathrm{L}$.

- tectorum L.

- virens Vill.

Dahlia variabilis Desf.

Dimorphotheca pluvialis

Moench.

Doronicum austriacum Jacq.

Echinops humilis. Bieb.

- Ritro L.

- sphaerocephalus L.

Erigeron acer $\mathrm{L}$.

- canadense L.

Fupatorium cannabinum L.

Gaillardia aristata Pursh.

Galinsoga parviflora L.

Helianthus annuus L.

- tuberosus L. (tub).

Fielichrysum arenarium DC.

- bracteatum Willd.

Hieracium aurantiacum L.

- bupleuroides Gmel.

- lanatum W. et K.

- pilosella L.

- sabaudum L.

- umbellatum $\mathrm{L}$.

- vulgatum Fries.

Hypochoeris glabra L.

- maculata L.

- radicata $L$.

Inula Helenium L.

Lactuca muralis Less.

- perennis L.

- saligna L.

- scariola L.

Lapsana communis $L$.

Leontopodium alpinum Cass.

Leontodon autumnalis L.

- hispidus L.

- hispidus L. v. hastilis

Madia sativa Mol.

Matricaria chamomilla L.

- discoidea DC.

Onopordon acanthium L.

Picris hieracioides $\mathrm{L}$.

Pulicaria vulgaris Gaert.

Pyrethrum roseum M. B.

Senecio paludosus $\mathrm{L}$.

- paluster Hook.

- silvaticus L.

- subalpinus Koch

- vulgaris L.

Scorzonera hispanica L.

- purpurea L. 
Serratula tinctoria L.

Silphium perfoliatum L.

Silybum Marianum Gaert.

Solidago virga-aurea $\mathrm{L}$.

Sonchus arvensis L.

- asper Hill.

- oleraceus L.

Tagetes signata Bartl.

Tanacetum vulgare $L$.

Taraxacum officinale Web.

Telekia speciosa Bmg.

Tragopogon dubius Scop.

- porrifolius L.

- pratensis L.

Xanthium italicum Mor.

- spinosum L.

Xeranthemum annuum L.

Commelinaceae.

Commelina coelestis Willd.

Tradescantia virginica $L$.

\section{Convolvulaceae.}

Calystegia sepium R. Br.

Convolvulus elongatus Willd.

- tricolor L.

Ipomea purpurea Lam.

- rubro-coerulea Hook.

Cuscuta epithymum L.

- europaea L.

- lupuliformis Krock.

\section{Crassulaceae.}

Sedum acre L.

- carpathicum Reuss.

- reflexum $\mathrm{L}$.

- spurium M. B.

Sempervivum soboliferum L.

\section{Cruciferae.}

Alliaria officinalis Andrz.

Alyssum calycinum L.

- montanum L.

- saxatile L. compactum

Arabis arenosa Scop.

- bellidifolia Jacq.

- hirsuta Scop.

Barbaraea vulgaris R. Br.
Barbaraea vulgaris R. Br. fol.

var.

Berteroa incana DC.

Brassica campestris L.

- nigra Koch

Bunias orientalis L.

Camelina microcarpa Andr'z.

- sativa Crantz

Capsella Bursa pastoris Mnch.

- Heegeri Solms

Cochlearia officinalis $\mathrm{L}$.

Cheiranthus cheiri L.

Coronopus Ruellii All.

Erysimum cheiranthoides L.

Hesperis matronalis $\mathrm{L}$.

- tristis L.

Iberis amara $\mathrm{L}$.

Isatis tinctoria $\mathrm{L}$.

Lepidium draba L.

- ruderale L.

- sativum L.

Lunaria annuua $\mathrm{L}$.

Myagrum perfoliatum L.

Nasturtium silvestre R. Br.

Raphanus sativus L.

Raphanus raphanistrum L.

Rapistrum perenne Berg.

Sinapis alba L.

- arvensis L.

Sisymbrium officinale Scop.

- sinapistrum Crantz

- Sophia L.

Stenophragma Thalianum Cel.

Thlaspi arvense L.

Turritis glabra L.

\section{Cucurbitaceae.}

Bryonia alba L.

- dioica Jacq.

\section{Cupressaceae.}

Biota orientalis Endl.

- orientalis Endl. var. pyramidalis Endl.

Chamaecyparis Lawsoniana

- nootkaensis Sudw. Parl.

Juniperus communis L.

- virginiana L.

Thuya gigantea Nutt. 
Thuya occidentalis $\mathrm{L}$.

- occidentalis L. aureo-spica

- occidentalis L. Wareana

hort.

\section{Cyperaceae.}

Carex acutiformis Ehrh.

- arenaria L.

- aristata R. Br. var. cuja-

- contigua Hoppe vica.

- distans L.

- flava L.

- Goodenoughii Gay

- gracilis Curt.

- hirta L.

- leporina L.

- panicea L.

- paniculata L.

- praecox Schreb.

- pseudocyperus L.

- posnaniensis Sprib.

- rostrata Stok.

- riparia Curt.

- secalina Wahlbg.

- teretiuscula Good.

- vesicaria L.

- vulpina L.

Cladium Mariscus R. Br. Eriophorum polystachium L. - vaginatum L.

Scirpus maritimus L. v. com-

- silvaticus L. pactus

- Tabernaemontani Gmel.

\section{Dipsacaceae.}

Cephalaria tatarica Schrad. - uralensis R. et Sch.

Dipsacus laciniatus L.

- pilosus L.

- silvester Huds.

Scabiosa columbaria L.

- lucida Vill.

- ochroleuca L.

\section{Eleagnaceae.}

Hippophae rhamnoides L.

\section{Ericaceae.}

Erica tetralix L.

Oxycoccos quadripetala Gilib.

Vaccinium uliginosum L.

\section{Euphorbiaceae.}

Euphorbia esula L.

- helioscopia L.

- Lathyris L.

- palustris L.

- peplus L.

Mercurialis annuua $\mathrm{L}$.

Ricinus communis $\mathrm{L}$.

\section{Fagaceae.}

Quercus borealis Michx. - robur L.

\section{Gentianacae.}

Gentiana asclepiadea L. - lutea L.

- pneumonanthe L.

- uliginosa Willd.

Sweertia alpestris Bmg.

\section{Geraniaceae.}

Erodium cicutarium L'Herit. Geranium pusillum Burm.

- sanguineum L.

\section{Gramineae.}

Agrostis vulgaris With.

- spica venti $L$.

Aira caespitosa L.

Alopecurus pratensis L.

Anthoxantum odoratum L.

Arrhenatherum elatius $M$. et

Koch

Brachypodium pinnatum P. B.

Briza maxima L.

- media L.

Bromus inermis Leyss.

- mollis L.

- secalinus L.

- sterilis L.

- tectorum L.

Calamagrostis arundinacea

— epigeios Rth. 
Calamagrostis lanceolata Rth.

Corynephorus canescens P. B. Dactylis Aschersoniana Graeb. - glomerata L.

Elymus arenarius L.

Eragrostis major Host.

- minor Host.

Festuca glauca Schrad.

- heterophylla Lmk.

- ovina L.

Glyceria aquatica Whlb.

- fluitans R. Br.

Holcus mollis L.

Hordeum murinum L.

Koeleria glauca L.

- gracilis DC.

Melica nutans L.

Milium effusum L.

Molinia coerulea Moench

Panicum crus galli L.

- miliaceum L.

- sanguinale L.

Phalaris arundinacea L.

Phleum Boehmeri Wib.

- pratense L.

Poa nemoralis L.

- pratensis L.

Stupa pennata L.

Triticum caninum $L$.

- spelta L.

\section{Guttiferae.}

Hypericum montanum I

- quadrangulum L.

- perforatum L.

\section{Iridaceae.}

Gladiolus imbricatus L.

Iris graminea $\mathrm{L}$.

- orientalis Thunb.

- pseudacorus L.

- sibirica L.

Sisyrinchium angustifolium

\section{Juglandaceae.}

Mill.

Carya cordiformis C. Koch

\section{Juncaceae.}

Juncus compressus Jacq. - conglomeratus L.
Juncus effusus $L$.

- glaucus Ehrh.

- lamprocarpus Ehrh.

Luzula nemorosa E. Meyer

- pallescens Bess.

- silvatica Gaud.

\section{Labiatae.}

Brunella grandiflora Jacq.

- vulgaris L.

Calamintha acinos Clairv.

- clinopodium Benth.

Dracocephalum Ruyschiana L.

- moldavicum L.

Elsholtzia Patrini Gcke

Galeopsis ladanum L.

- pubescens Bess.

Hyssopus officinalis L.

Lamium album L.

- amplexicaule L.

Leonurus cardiaca L.

Lycopus europaeus L.

Marrubium vulgare L.

Melissa officinalis L.

Mentha arvensis L.

- pulegium L.

- silvestris L.

Monarda fistulosa L.

Nepetha cataria L.

- Mussini Spreng.

Origanum majorana L.

- vulgare L.

Phlomis alpina Pall.

Salvia argentea L.

- cleistogama De Barry et

- Hormium Batt.

Paul.

- - v. rubra

- glutinosa L.

- officinalis L.

- pratensis L.

- silvestris L.

- verticillata L.

Satureja hortensis L.

Scutellaria altissima $\mathrm{L}$.

Stachys lanatus Jacq.

- officinalis Trev.

- palustris L.

- rectus $\mathrm{L}$. 
Stachys silvaticus L.

Teucrium chamaedrys L. - scorodonia L.

Thymus serpyllum Borb.

- sudeticus Borb.

\section{Liliaceae.}

Allium fistulosum L.

- montanum Schm.

- schoenoprasum L.

- scorodoprasum L. (bulb.)

- vineale L. (bulb.)

Anthericum liliago L.

- ramosum L.

Asparagus ofiicinalis L.

Colchicum autumnale L.

Fritillaria imperialis L.

Hemerocallis fulva L.

Lilium bulbiferum L. (bulb.)

- martagon L.

- tigrinum (bulb.)

Scilla bifolia L.

- sibirica Andrz.

Tofieldia calyculata Wahlbs.

\section{Linaceae.}

Linum grandiflorum L.

- perenne L.

- usitatissimum L.

\section{Loasaceae.}

Blumenbachia Hieronymii Urb.

Loasa vulcanica André

\section{Lythraceae.}

Lythrum hyssopifolia L.

- salicaria L.

\section{Malvaceae.}

Althaea officinalis L.

- rosea Cav.

- - - v. nigra

Lavatera thuryngiaca $\mathrm{L}$.

Malope trifida Cav.

Malva alcea L.

Malva crispa L.

- moschata L.

- rotundifolia $\mathrm{L}$.

- silvestris L.

\section{Oenotheraceae.}

Epilobium collinum Gmel.

- hirsutum L.

- montanum L.

- roseum Schreb.

\section{Oleaceae.}

Fraxinus americana $L$.

- exelsior L.

Ligustrum brachystachium

Decn.

- vulgare L.

Syringa vulgaris $\mathrm{L}$.

\section{Oxalidaceae.}

Oxalis corniculata L. atropurp. - stricta L.

\section{Papaveraceae.}

Adlumia cirrhosa Rafin. Bocconia cordata Willd. Chelidonium majus L.

- - L. laciniatum

Eschscholzia californica Cham. Fumaria officinalis L.

- Vaillantii Lois.

Glaucium flavum Crantz

Papaver argemone L.

— dubium L.

- orientale L.

- rhoeas L.

- - L. flore pleno

- somniferum L.

- L. flore pleno

- - L. mostrosum

\section{Papilionaceae.}

Amorpha canescens Nutt. Anthyllis Kerneri Sag. Astragalus arenarius L.

- cicer L.

- glycyphyllos L.

- penduliflorus Lam.

Caragana arborescens Lam.

Colutea arborescens L.

Cytisus ratisbonensis Schäff.

Galega officinalis L.

Genista tinctoria L.

Laburnum alpinum Ber. et

Presl. 
Laburnum anagyroides Med.

— vulgare Gris.

- - Gris. v. quercifolium

Lathyrus maritimus Big.

- niger Bernh.

- odoratus L. hybr.

- vernus Bernh.

Lotus siliquosus L.

- uliginosus Schkuhr

Lupinus angustifolius $\mathrm{L}$.

- polyphyllus L.

- varius L.

Medicago falcata L.

- lupulina L.

- sativa $L$.

Melilotus albus Desr.

- dentatus Pers.

- officinalis Med.

Onobrychis viciaefolia Scop.

Ononis spinosa L.

Phaseolus multiflorus L.

Pisum arvense $\mathrm{L}$.

Trifolium alpestre L.

- arvense L.

- aureum Poll.

- fragiferum L.

- hybridum L.

- lupinaster L.

- montanum L.

- repens L.

- resupinatum L.

- rubens L.

Vicia cassubica L.

- cracca L.

- dumetorum L.

- hirsuta Koch

- pisiformis L.

- sepium L.

- tetrasperma Mnch.

- villosa Rth.

\section{Phytolaccaceae.}

Phytolacca decandra L.

\section{Pinaceae.}

Abies cephalonica Lk.

- Nordmanniana Lk.

- sibirica Ledeb.

Picea alba Lk.
Picea orientalis Carr.

Pinus nigra Arn.

- rigida Mill.

- strobus L.

Tsuga canadensis Carr.

Plantaginaceae.

Plantago cynops L.

- lanceolata L.

- major L.

- - L. var. monstrosa

- - L. var. rubrifolia

- maritima L.

- maxima Ait.

- media L.

- ramosa Aschers.

\section{Platanaceae.}

Platanus acerifolia Willd.

\section{Plumbaginaceae.}

Armeria vulgaris Willd.

Limonium Bonduellei O. Ktze.

Polemoniaceae.

Collomia grandiflora Dougl.

- linearis Nutt.

Polemonium coeruleum L.

\section{Polygalaceae.}

Polygala comosa Schkuhr

\section{Polygonaceae.}

Fagopyrum tataricum Gaert. Polygonum aviculare L.

- bistorta L.

- convolvulus L.

- hydropiper L.

- nodosum Pers.

- persicaria L.

- viviparum L.

Rheum palmatum $\mathrm{L}$.

- undulatum L.

Rumex acetosa L.

- acetosella L.

- conglomeratus Murr.

- crispus L.

- hydrolapathum Huds.

- patientia L. 
Rumex scutatus L.

- silvestris Wallr.

\section{Portulacaceae.}

Claytonia perfoliata Don. Portulaca oleracea L.

\section{Primulaceae.}

Anagalis arvensis L.

- coerulea Schreb.

Androsace septentrionalis L.

Lysimachia vulgaris $L$.

Primula cortusoides L.

- officinalis Jacq.

- japonica A. Gray

\section{Ranunculaceae.}

Aconitum Napellus L.

Actaea spicata L.

Adonis aestivalis L.

- vernalis $L$.

Aquilegia canadensis L.

- chrysantha A. Gray.

- Helenae hort.

- vulgaris L.

Caltha palustris L.

Clematis campaniflora Brot.

- integrifolia L.

- serratifolia Rehd.

- tangutica Andr.

- vitalba $L$.

- viticella L.

Delphinium ajacis L.

- consolida L.

- elatum L.

- oxysepalum Pax et Borb.

Myosurus minimus L.

Nigella sativa L.

Pulsatilla patens Mill.

- pratensis Mill.

Ranunculus acer L.

- aconitifolius L.

- arvensis L.

- auricomus L.

- bulbosus L.

- Flamula L.

- polyanthemos L.

- repens $\mathrm{L}$.

- sardous $\mathrm{Cr}$
Ranunculus sceleratus L. Villarsii DC.

Thalictrum angustifolium L.

- aquilegifolium L.

- minus L.

- simplex L.

Trollius europaeus L.

\section{Resedaceae.}

Reseda luteola L.

- phyteuma L.

\section{Rhamnaceae.}

Rhamnus cathartica L. - frangula L.

\section{Rosaceae.}

Agrimonia eupatoria L.

- odorata Mill.

Alchemilla pubescens Lam.

- silvestris Schm.

Aruncus silvester Kost.

Comarum palustre L.

Cotoncaster horizontalis Decn.

- melanocarpa Lood.

Crataegus crus galli L.

- Oxyacantha L.

- sanguinea Pall.

Filipendula hexapetala Gilib.

- ulmaria Max.

Fragaria vesca L. var. mono-

Geum rivale L.

phylla

- urbanum L.

- urb. $\times$ G. rivale

Potentilla argentea L.

- aurea L.

- procumbens Sibth.

- recta $L$.

- rupestris $\mathrm{Cr}$.

Physocarpus opulifolius Max. Rhodotypus kerrioides S. et Z. Rosa multiflora Thunb.

- rubiginosa $\mathrm{L}$.

- rubrifolia Vill.

- rugosa Thunb.

Sanguisorba minor Scop.

- officinalis L. 


\section{Rubiaceae.}

Asperula tinctoria L.

Galium anisophyllum Vill.

- aparine L.

- boreale L.

- mollugo L.

- Schulthesii Vest..

- silvaticum L.

- vernum Scop.

Rubia tinctorum L.

Sherardia arvensis L.

\section{Rutaceae.}

Dictamnus albus L. Ruta graveolens L.

\section{Saxifragaceae.}

Saxifraga Aizoon Jacq. - granulata L.

\section{Scrophulariaceae.}

Alectorolophus major Rchb. Antirrhinum orontium L. Digitalis ambigua Murr.

- lutea L.

- purpurea L.

- purpurea L. peloria Gratiola officinalis L.

Linaria elatine Mill. - genistifolia Mill.

Pedicularis silvatica $L$. - verticillata L.

Pentstemon alpinus Torr.

- azureus

- barbatus Roth.

Scrophularia alata Gilib.

- nodosa L.

Veronica beccabunga L.

- hederifolia L.

- longifolia L.

- officinalis L.

- orchidea Cr.

- serpyllifolia L.

- spicata L.

- teucrium L.

Verbascum lychnitis L.

- nigrum L.

- phoeniceum L.

- phlomoides L.
Verbascum speciosum Schrad. - thapsus L.

\section{Solanaceae.}

Atropa Belladonna L.

Datura stramonium L.

- inermis Jacq.

- Tatula L.

Hyoscyamus niger L.

Nicandra physaloides Gaert.

Nicotiana rustica $L$.

- tabacum L.

Petunia nyctaginiflora Juss.

Physalis Alkekengi L.

Solanum dulcamara L.

- luteum Mill.

- nigrum L.

\section{Sparganiaceae.}

Sparganium minimum Fries.

- simplex Huds.

Tiliaceae.

Tilia argentea Desf.

- cordata Mill.

- intermedia DC.

\section{Tropacolaceae.}

Tropaeolum majus $\mathrm{L}$.

- majus L. fol. var.

\section{Umbelliferae.}

Aegopodium podagraria L.

Aethusa cynapium L.

Anethum graveolens L.

Angelica silvestris L.

Anthriscus cerefolium Hoffm.

- silvester Hofim.

Archangelica officinalis Hoffm.

Astrantia major L.

Carum Carvi L.

Chaerophyllum aromaticum $\mathrm{L}$.

- temulum L.

Cicuta virosa $\mathrm{L}$.

Conium maculatum L.

Coriandrum sativum L.

Daucus carota L.

Eryngium campestre L.

- maritimum L. 
Eryngium planum L.

Falcaria vulgaris Bernh.

Foeniculum vulgare Mill. Hacquetia epipactis Neck. Heracleum sibiricum L. - sphondylium L.

Levisticum officinale $L$. Libanotis montana Crantz Oenanthe Phellandryum DC.

Pastinaca sativa L.

Peucedanum cervaria Cuss.

- Oreoselium Mnch.

- palustre Moench

Pimpinella major L. lacin.

- saxifraga L.

Scandix pecten Vencris L.

Torilis Anthriscus Gmel.

\section{Urticaceae.}

Parietaria officinalis $L$. Urtica cannabina $L$.
Urtica dioica L.

- pilulifera L.

Valerianaceae.

Valeriana officinalis L.

Valerianella olitoria Poll.

\section{Verbenaceae.}

Verbena hybrida hort. grdfl.

- officinalis L.

\section{Violaceae.}

Viola arvensis Murr.

- canina Rchb.

- elatior Fr.

\section{Vitaceae.}

Ampelopsis quinquefolia Mchx. Vitis riparia Mchx.

Posnaniae, Januarii 1929.

Prof. Dr. A. Wodziczko horti botan. director.
J. W. Szulczewski adjunctus.

W1. Drzewiecki

horti botan. inspector. 

Hortus Botanicus Posnaniensis

Poznań (Polonia)

Dąbrowskiego 165.

000

\section{SELECTUS SEMINUM \\ ANNO 1929LCOLLECTORUM \\ QUAE HORTUS BOTANICUS POSNANIENSIS \\ MUTUO COMMUTANDA OFFERT.}

DESIDERATA ANTE DIEM 1. III. 1930 NOBIS COMMUNICANDA SUNT.

Aceraceae.

Acer campestre L.

- negundo $\mathrm{L}$.

- plantanoides L.

\section{Alismataceae.}

Alisma plantago L.

\section{Amarantaceae.}

Amarantus ascendens Loiss.

- caudatus L.

- caudatus L. albiflorus

- paniculatus L.

- retroflexus L.

Anacardiaceae.

Rhus typhina L.

\section{Araceae.}

Calla palustris ' $\mathrm{L}$.

\section{Asclepiadaceae.}

Asclepias Cornuti Decn.

Vincetoxicum nigrum Moench

- officinale Moench

\section{Berberidaceae.}

Berberis Thunbergi DC.

- vulgaris L.

- vulgaris L. f. atropurp.

Betulaceae.

Alnus glutinosa Gaert.

- incana Moench
Betula humilis L.

- lenta Lia

- nana L.

- oycoviensis Bess.

- papyracea Ait.

- pubescens Ehrh.

- verrucosa Ehrh.

Borragínaceae.

Anchusa officinalis L.

Asperugo procumbens L .

Cerinthe minor $\mathrm{L}$.

Cynoglossum officinale L.

Echium vuloare L.

Lappula echinata Gilib.

Lithospermum officinale L.

Lycopsis arvensis L.

Symphytum officinale L.

\section{Campanulaceae.}

Adenophora Potanini Fisch.

Campanula bononiensis L.

- carpatica Jacq.

- cervicaria L.

- glomerata L.

- Kladniana Schur.

- medium L.

- rapunculoides $\mathrm{L}$.

- sibirica L.

- trachelium L.

Jasione montana L

Phyteuma orbiculare I

Specularia Specalum 
Caprifoliaceae.

Lonicera sempervirens L. cocc. superba

Symphoricarpus racemosus

Viburnum opulus L.

Michx.

\section{Caryophyllaceae.}

Agrostemma githago L. Arenaria serpyllifolia L. Cerastium tomentosum L. Cucubalus baccifer L. Dianthus Armeria L.

- barbatus L.

- caesius Sm.

- carthusianorum L.

- deltoides L.

- plumarius L.

- praecox W. K.

- Seguieri Vill.

Gypsophila paniculata L. Heliosperma quadrifida Rchb. Holosteum umbellatum L. Lychnis coronaria Desv. - flos cuculi L.

Melandryum album Gcke.

- noctiflorum Fries.

- rubrum Gcke.

- Zawadzkii A. Br. Saponaria officinalis L. Silene conica L.

- venosa Aschers.

Spergula arvensis L.

Stellaria media Cyr.

Tunica prolifera Scop.

- saxifraga Scop.

Viscaria vulgaris Röhl.

\section{Celastraceae.}

Evonymus alata C. Koch.

- europaea L.

- verrucosa Scop.

\section{Chenopodiaceae.}

Atriplex hortense L.

- patulum L.

Chenopodium album $\mathrm{L}$.

- ambrosioides L.

- bonus Henricus L.

- hybridum L.

- polyspermum L.
Salsola Kali L.

Spinacia oleracea L.

\section{Cístaceae.}

Helianthemum polifolium DC.

\section{Compositae.}

Achillea distans W. K.

- millefolium L.

Ammobium alatum $\mathrm{R}, \mathrm{Br}$.

Anthemis nobilis L.

- ruthenica M. B.

Arctium lappa L.

- minus Bernh.

- nemorosum Lej.

- tomentosum Mill.

Arnica montana L.

Artemisia absinthium L.

- campestris L.

- vulgaris L.

Aster alpinus L.

Bellis perennis L.

Bidens cernuus $\mathrm{L}$.

- tripartitus L.

Calendula arvensis L.

- officinalis L.

Carduus acanthoides L.

- nutans L.

Carlina acaulis L. var. caules-

Carthamus tinctorius $\mathrm{L}$.

cens.

Centaurea cyanus L.

- Kotschyana Heuff.

T oxylepis Hay.

f? ruthenica Lam.

- rhenana Bor.

- scabiosa L.

- serotina Bor.

- sulphurea Bor.

Chamaepeuce diacantha DC.

Chrysanthemum carinatum

- leucanthemum L.

Schousb.

- segetum L.

Cirsium acaule All.

- arvense Scop.

- canum Bieb.

- eriophoum Scop.

- erisithales Scop.

- lanceolatum Scop. 
Cirsium palustre Scop.

- acaule All. $X$ oleraceum Scop.

Coreopsis tinctoria Nutt. - - Nutt. v. prolifica Rac.

- grandiflora Nutt.

Cosmos bipinnatus Cav.

Crepis biennis L.

- tectorum L.

- virens Vill.

Dimorphotheca aurantiaca DC. - pluvialis Moench.

Echinops Ritro L.

- sphaerocephalus L.

Erigeron acer L.

- canadense L.

Eupatorium cannabinum L.

Filago arvensis L.

Gnaphalium silvaticum L.

Helianthus annuus L. var. Bismarckianus.

- argyrophyllus Torr, et

- tuberosus L. (tub).

\section{Gray.}

Hieracium alpinum $\mathrm{L}$.

- aurantiacum L.

- bupleuroides Gmel.

- lanatum W. et $K$.

- pilosella L.

- sabaudum L.

- umbellatum L.

- vulgatum Fries.

Hypochoeris glabra L.

- radicata $L$.

Inula Helenium L.

- salicina L.

Lactuca muralis Less.

- perennis L.

- saligna L.

- scariola L.

Lapsana communis L.

Leontopodium alpinum Cass.

Leontodon autumnalis L.

- hispidus L.

- hispidus L. v. hastilis.

Madia sativa Mol.

Matricaria chamomilla L.

- discoidea DC.

- inodora L.

Mulgedium alpinum Cass.
Onopordon acanthium L.

Pulicaria vulgaris Gaert.

Saussurea macrophylla Saut.

Senecio adonidifolius Loisel.

- paludosus L.

- paluster Hook.

- silvaticus L.

- subalpinus Koch.

- vernalis W. K.

- vulgaris L.

Scorzonera hispanica L.

- purpurea L.

Serratula tinctoria L.

Silphium perfoliatum L.

Silybum Marianum Gaert.

Solidago virga-aurea L.

Sonchus arvensis L.

- asper Hill.

- oleraceus L.

Tagetes signata Bartl.

Tanacetum vulgare $L$.

Taraxacum officinale Web.

Telekia speciosa Bmg.

Tragopogon dubius Scop.

- porrifolius L.

- pratensis L.

Xanthium italicum Mor,

- spinosum L.

\section{Commelinaceae.}

Commelina coelestis Willd. Tradescantia virginica $\mathrm{L}$.

\section{Convolvulaceae.}

Calystegia sepium R. Br.

Convolvulus arvensis $\mathrm{L}$.

- elongatus Willd.

- tricolor L.

Cuscuta epilinum Weihe.

- lupuliformis Krock.

Ipomea purpurea Lam.

- rubro-coerulea Hook.

Cornus alba L.

\section{Cornaceae.}

Crassulaceae.

Sedum acre L.

- carpathicum Reuss.

- maximum Sut.

- polonicum Bl. 
Sedum reflexum $\mathrm{L}$.

- spurium M. B.

Sempervivum soboliferum L. (bulb.)

\section{Cruciferae.}

Alliaria officinalis Andrz.

Arabis arenosa Scop.

- bellidifolia Jacq.

- hirsuta Scop.

Barbaraea vulgaris $\mathrm{R}$. Br.

- vulgaris $\mathrm{R}$. Br. fol. var.

Biscutella laevigata L.

Brassica campestris L.

- nigra Koch.

Bunias orientalis L.

Cakile maritima Scop.

Camelina microcarpa Andrz.

- sativa Crantz.

Capsella Bursa pastoris Mnch.

Coronopus Ruellii All.

Diplotaxis tenuifolia DC.

Erysimum cheiranthoides L.

Hesperis matronalis L.

- tristis L.

Iberis amara $\mathrm{L}$.

Lepidium draba $\mathrm{L}$.

- ruderale $\mathrm{L}$.

- sativum L.

Lunaria annuua $\mathrm{L}$.

Myagrum perfoliatum L.

Nasturtium silvestre R. Br.

Raphanus sativus L.

Raphanus raphanistrum L.

Sinapis alba L.

- arvensis L.

Sisymbrium officinale Scop.

- sinapistrum Crantz.

- Sophia L.

Stenophragma Thalianum Cel.

Thlaspi arvense $\mathrm{L}$.

Turritis glabra L.

\section{Cucurbitaceae.}

Bryonia alba L.

- dioica Jacq.

Cucurbita Pepo L.

- - v. turbaniformis.

\section{Cupressaceae.}

Chamaecyparis nootkaensis

Sudw.
Thuya occidentalis L.

- occidentalis L. aureo-spica

Cyperaceae.

Carex contigua Hoppe.

- distans L.

- flava L.

- hirta L.

- leporina L.

- panicea L.

- paniculata L.

- pseudocyperus L.

- secalina Wahlbg.

- teretiuscula Good.

- vesicaria $\mathrm{L}$.

- vulpina $\mathrm{L}$.

Cladium Mariscus R. Br.

Eriophorum polystachium L.

- vaginatum $\mathrm{L}$.

Scirpus compressus Pers.

- lacustris L.

- maritimus L. v. compactus.

- silvaticus L.

- Tabernaemontani Gmel.

\section{Dipsacaceae.}

Cephalaria uralensis $\mathrm{R}$, et Sch.

Dipsacus laciniatus L.

- pilosus L.

- silvester Huds.

Scabiosa columbaria L.

- lucida Vill.

- maritima L. var. atropurpurea prolifica.

- ochroleuca L.

Succissa pratensis Mnch.

Eleagnaceae.

Hippophae rhamnoides L.

Empetraceae.

Empetrum nigrum $\mathrm{L}$.

Euphorbiaceae.

Mercurialis annuua L.

Ricinus communis L.

Gentianaceae.

Gentiana asclepiadea L.

- cruciata L.

Sweertia alpestris Bmg. 


\section{Geraniaceae.}

Erodium cicutarium L'Herit.

Geranium pratense L.

- pusillum Burm.

- sanguineum L.

- silvaticum L.

\section{Gramineae.}

Agrostis spica venti $L$.

Alopecurus pratensis L.

Brachypodium pinnatum P. B.

Briza media L.

Bromus inermis Leyss.

- mollis L.

- secalinus L.

- sterilis L.

- tectorum L.

Dactylis glomérata L.

Elymus arenarius L.

Eragrostis major Host,

- minor Host.

- pilosa P. B.

Festuca elatior L.

Koeleria glauca L.

- gracilis DC.

Melica nutans L.

Panicum crus galli $\mathrm{L}$.

Phleum Boehmeri Wib.

- pratense L.

Setaria glauca P. B.

- viridis P. B.

Stupa capillata L.

- pennata L.

Triticum caninum $\mathrm{L}$.

- spelta L.

\section{Hydrophyllaceae.}

Phacelia tanacetifolia Benth.

\section{Iridaceae.}

Gladiolus imbricatus L.

Iris sibirica $\mathrm{L}$.

Sisyrinchium angustifolium Mill.

- anceps Cav.

\section{Juncaceae.}

Juncus compressus Jacq.

- conglomeratus L.

- effusus L.

- - var. zebrinus Hort.
Juncus glaucus Ehrh.

Luzula silvatica Gaud.

\section{Juncaginaceae.}

Triglochin maritimum L.

- palustre L.

\section{Labiatae.}

Brunella grandiflora Jacq.

- vulgaris L.

Calamintha acinos Clairv.

- clinopodium Benth.

Dracocephalum Ruyschiana L.

Elsholtzia Patrini Gcke.

Galeopsis bifida Boenn.

- ladanum L.

- speciosa Mill.

Hyssopus officinalis L.

Leonurus cardiaca L.

Lycopus europaeus L.

Marrubium vulgare $\mathrm{L}$.

Melissa officinalis L.

Mentha arvensis L.

- silvestris L.

Monarda fistulosa L.

Nepetha cataria L.

Origanum vulgare $\mathrm{L}$.

Phlomis alpina Pall.

Salvia argentea $\mathrm{L}$.

- Hormium Batt.

- - v. rubra.

- glutinosa L.

- officinalis L.

- pratensis L.

- silvestris L.

- verticillata $\mathrm{L}$.

Scutellaria altissima L.

- galericulata L.

Stachys germanica L.

- lanatus Jacq.

- officinalis Trev.

- palustris L.

- rectus L.

- silvaticus L.

Teucrium chamaedrv; I.

- montanum L, var, pannonicum Kern.

- scorodonia L.

Thymus ovatus Mill.

- sudeticus Borb. 


\section{Liliaceae.}

Allium fistulosum $\mathrm{L}$.

- montanum Schm.

- schoenoprasum L.

- scorodoprasum L. (bulb.)

- vineale L. (bulb.)

Anthericum liliago L.

- ramosum L.

Asparagus officinalis L.

Colchicum autumnale L.

Fritillaria imperialis L.

- Meleagris L.

Galtonia candicans Dene.

Hemerocallis flava $L$. fulva $L$.

Lilium bulbiferum L. (bulb.)

- martagon L.

- tigrinum Ker-Gawl. (bulb.)

Polygonatum multiflorum All.

- verticillatum All.

Scilla sibirica Andrz.

Tofieldia calyculata Wahlbg.

Tulipa silvestris L.

\section{Linaceae.}

Linum perenne L.

- usitatissimum L.

\section{Lentibulariaceae.}

Pinguicula vulgaris $L$.

\section{Loasaceae.}

Bartonia aurea Lindl.

Blumenbachia Hieronymi Urb.

\section{Lythraceae.}

Lythrum hyssopifolia L.

- salicaria L.

\section{Malvaceae.}

Althaea officinalis L.

- rosea Cav.

- - v vigra.

Lavatera thuryngiaca $\mathrm{L}$.

Malope trifida Cav.

Malva moschata L.

- neglecta Wallr.

- rotundifolia L.

- silvestris L.

\section{Oenotheraceae.}

Epilobium angustifolium L.

- hirsutum L.

- montanum L.

- roseum Schreb.

Oenothera biennis L.

\section{Oleaceae.}

Fraxinus exelsior L.

- - var. pendula.

Ligustrum vulgare $\mathrm{L}$.

- - var. xanthocarpum.

Syringa vulgaris $\mathrm{L}$.

\section{Oxalidaceae.}

Oxalis corniculata L.

- - var, atropurp.

- stricta L.

\section{Papaveraceae.}

Chelidonium majus L.

- - L. laciniatum.

Corydalis cava Schwg. et K.

Eschscholzia californica Cham.

Fumaria officinalis L.

- Vaillantii Lois.

Glaucium flavum Crantz.

Papaver argemone L.

- dubium L.

- orientale L.

- rhoeas L.

- somniferum L.

\section{Papilionaceae.}

Amorpha canescens Nutt.

Anthyllis Kerneri Sag.

Astragalus glycyphyllos L.

Caragana frutescens DC.

Cytisus ratisbonensis Schäff.

Galega officinalis L.

Genista sagittalis L.

- tinctoria L.

Lathyrus latifolius L.

- maritimus Big.

- montanus Bernh.

- niger Bernh.

- odoratus L. hybr.

- pratensis L.

- silvester L.

- vernus Bernh. 
Lotus siliquosus L.

Lupinus angustifolius L.

- luteus L.

- polyphyllus L.

Medicago falcata $\mathrm{L}$.

- lupulina L.

- sativa L.

Melilotus albus Desr.

- dentatus Pers.

- officinalis Med.

Onobrychis viciaefolia Scop.

Ononis hircina Jacq.

- spinosa L.

Phaseolus multiflorus L.

Pisum arvense $\mathrm{L}$.

Robinia neo-mexicana Gray.

- Pseudacacia L.

Trifolium alpestre $\mathrm{L}$.

- arvense L.

- fragiferum L.

- repens L.

Trigonella coerulea Ser.

Vicia cassubica L.

- cracca L.

- dumetorum L.

- Faba L.

- hirsuta Koch.

- sepium L.

- villosa Rth.

Picea alba Lk.

Pinus montana Mill.

- silvestris L.

\section{Plantaginaceae.}

Plantago cynops $\mathrm{L}$.

- lanceolata L.

- major L.

- - L. var. monstrosa.

- media L.

- ramosa Aschers. .

Plumbaginaceae.

Armeria vulgaris Willd.

Statice Bonduelli Lestib.

- latifolia Smith.

\section{Polemoniaceae.}

Collomia grandiflora Doug1. Polemonium coeruleum L.

\section{Polygonaceae.}

Fagopyrum tataricum Gaert.

Polygonum aviculare L.

- bistorta L.

- convolvulus L.

- dumetorum L.

- nodosum Pers.

- persicaria L.

- viviparum L.

Rheum palmatum L.

- undulatum L.

Rumex acetosa L.

- conglomeratus Murr.

- crispus L.

- hydrolapathum Huds.

- maritimus L.

- patientia L.

- scutatus L.

- silvestris Wallr.

\section{Portulacaceae.}

Claytonia perfoliata Don.

Portulaca oleracea L.

\section{Primulaceae.}

Anagalis arvensis L.

Androsace lactea L.

- septentrionalis L.

- lactea L.

Lysimachia punctata L.

- vulgaris L.

Primula elatior Hill.

- officinalis Jacq.

\section{Ranunculaceae.}

Actaea spicata L.

Adonis aestivalis $\mathrm{L}$.

- autumnalis L.

- vernalis L.

Aquilegia chrysantha A. Gray.

- haylodgensis Hort.

- Helenae hort.

- vulgaris $\mathrm{L}$.

- - var. duplex.

- var. fl. pleno.

Anemone silvestris $L$.

Caltha palustris L.

Clematis integrifolia $\mathrm{L}$.

- recta $\mathrm{L}$.

- vitalba $\mathrm{L}$.

- viticella L. 
Delphinium ajacis L.

- chinense Fisch.

- consolida L.

- elatum L.

Myosurus minimus L.

Nigella damascena L.

- - fl. pleno.

- sativa $\mathrm{L}$.

Pulsatilla patens Mill.

Ranunculus acer $\mathrm{L}$.

- aconitifolius L.

- arvensis L.

- auricomus L.

- lanuginosus L.

- Lingua L.

- polyanthemos L.

- repens L.

- sardous Cr.

- sceleratus L.

- Villarsii DC.

Thalictrum angustifolium $\mathrm{L}$.

- aquilegifolium L.

- minus L.

Trollius europaeus $L$.

- transsilvanicus Schur.

Reseda luteola L.

Resedaceae.

- phyteuma L.

\section{Rhamnaceae.}

Rhamnus cathartica L.

- frangula $\mathrm{L}$.

\section{Rosaceae.}

Agrimonia eupatoria L.

Comarum palustre L.

Cotoneaster acutifolia Turcz.

- integerrima Medic.

- melanocarpa Lood.

Crataegus monogyna Jacq.

Filipendula hexapetala Gilib.

- ulmaria Max.

Geum rivale $\mathrm{L}$.

- urb. $\times$ G. rivale.

Potentilla argentea L.

- aurea L.

- procumbens Sibth.

- recta $L$.

- rupestris $\mathrm{Cr}$.

Physocarpus opulifolius Max.
Rosa canina L.

- pendulina L.

- rubiginosa $\mathrm{L}$.

- rubrifolia Vill.

- tomentosa Sm.

Sanguisorba minor Scop.

- officinalis L.

Sorbus aucuparia L.

\section{Rubiaceae.}

Asperula tinctoria L.

Galium aparine L.

- boreale L .

- mollugo L.

- Schulthesii Vest.

Sherardia arvensis L.

Rutaceae.

Dictamnus albus L.

Ruta graveolens L.

\section{Saxifragaceae.}

Saxifraga granulata L.

\section{Scrophulariaceae.}

Alectorolophus major Rchb.

Antirrhinum orontium L.

Digitalis ambigua Murr.

- lanata Ehrh.

- lutea L.

- purpurea L. peloria.

- lan. Ehrh. X lutea L.

Gratiola officinalis L.

Linaria elatine Mill.

- genistifolia Mill.

- vulgaris Mill.

Scrophularia alata Gilib.

- nodosa L.

- Scopolii Hoppe.

Veronica beccabunga L.

- chamaedrys L.

- hederifolia L.

- longifolia L.

- officinalis L.

- orchidea $\mathrm{Cr}$.

- saxatilis Scop.

- serpyllifolia L.

- spicata L.

- teucrium L.

Verbascum lychnitis L. 
Verbascum nigrum L.

- phoeniceum L.

- phlomoides L.

- thapsus L.

- thapsiforme Schrad.

\section{Solanaceae.}

Datura stramonium L.

- inermis Jacq.

- Tatula L.

Hyoscyamus niger L.

Nicandra physaloides Gaert.

Nicotiana paniculata L.

- rustica L.

Physalis Alkekengi L.

Solanum dulcamara L.

- luteum Mill.

- nigrum L.

\section{Sparganiaceae.}

Sparganium minimum Fries.

- simplex Huds.

\section{Tamaricaceae.}

Myricaria germanica Desv.

\section{Tiliaceae.}

Tilia argentea Desf.

- cordata Mill.

- intermedia DC.

- spectabilis Dipp.

\section{Tropaeolaceae.}

Tropaeolum majus L.

- majus L. fol, var.

\section{Umbelliferae.}

Aegopodium podagraria L.

Aethusa cynapium L.

Anethum graveolens L.

Angelica silvestris L.

Anthriscus silvester Hoffm.

Archangelica officinalis Hoffm.

Astrantia major L.

Berula angustifolia Koch.

Carum Carvi L.

Chaerophyllum aromaticum $\mathrm{L}$.
Chaerophyllum temulum $\mathrm{L}$.

Cicuta virosa $L$.

Conium maculatum L.

Coriandrum sativum $\mathrm{L}$.

Daucus carota L.

Eryngium campestre L.

- maritimum L.

- planum L.

Heracleum Mantegazzianum

- sibiricum L.

Som. et Lev.

- sphondylium L.

Laserpitium latifolium L.

Levisticum officinale $\mathrm{L}$.

Libanotis montana Crantz.

Oenanthe Phellandryum DC.

Pastinaca sativa L.

Peucedanum cervaria Cuss.

- Oreoselium Mnch.

- palustre Moench.

Scandix pecten Veneris L.

Seseli rigidum $W$. et $K$.

Torilis Anthriscus Gmel.

Urticaceae.

Parietaria officinalis $\mathrm{L}$.

Urtica cannabina L.

- dioica L.

- pilulifera L.

Valerianaceae.

Valeriana officinalis L.

Verbenaceae.

Verbena hybrida hort. grdfl.

- officinalis L.

- venosa Gill, et Hook.

\section{Violaceae.}

Viola arvensis Murr.

- canina Rchb.

- declinata W. K.

- elatior Fr.

- Riviniana Rchb.

- silvestris Rchb.

\section{Vitaceae.}

Ampelopsis quinquefolia Mchx.

Posnania e, Januarii 1930.

Prof. Dr. A. Wodziczko

horti botan. director.
J. W. Szulczewski

adjunctus.
W1. Drzewiecki

horti botan. inspector. 


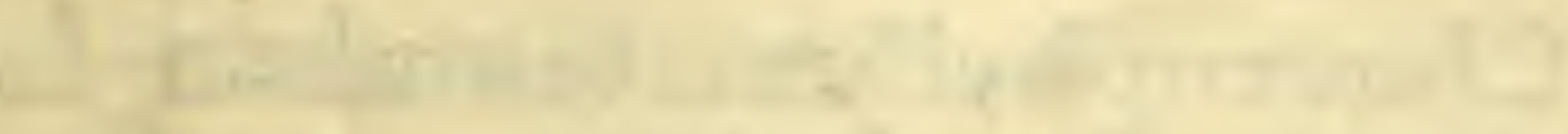

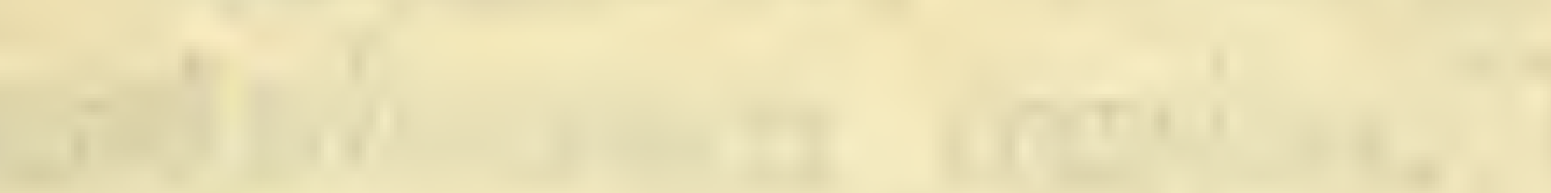

-

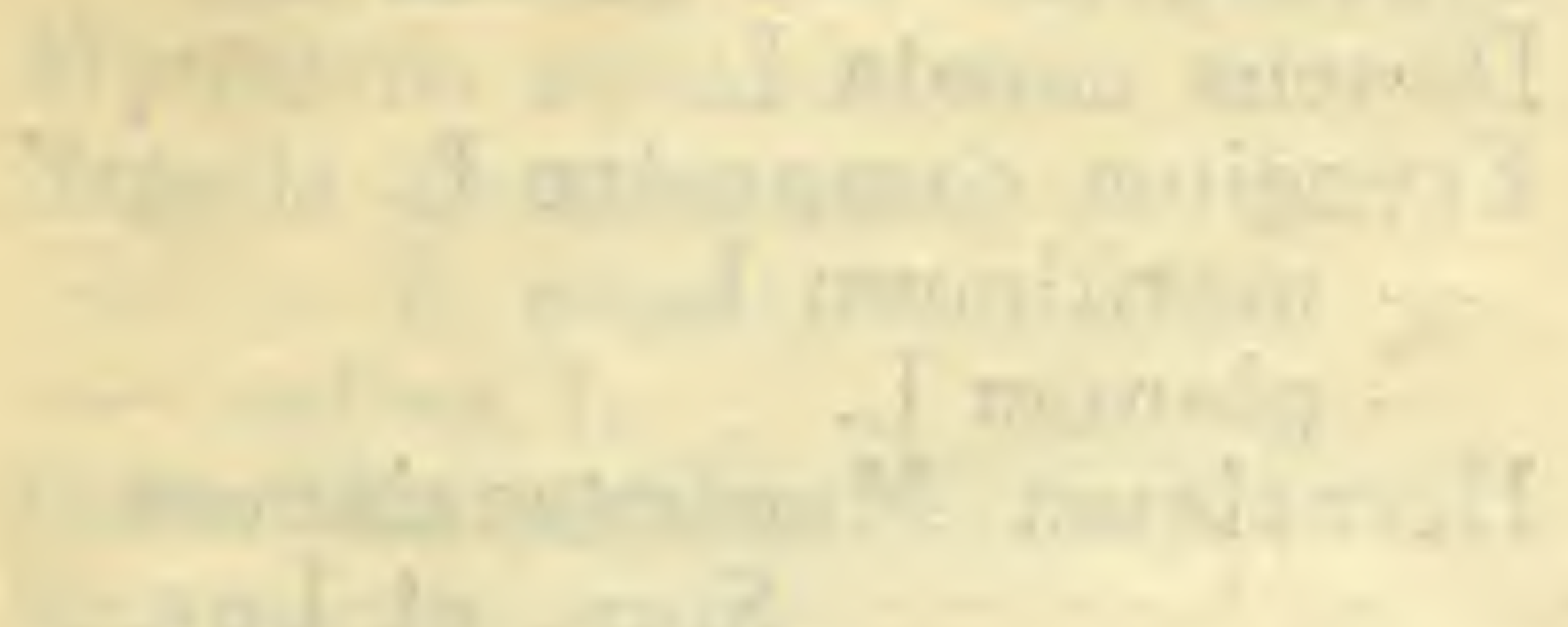

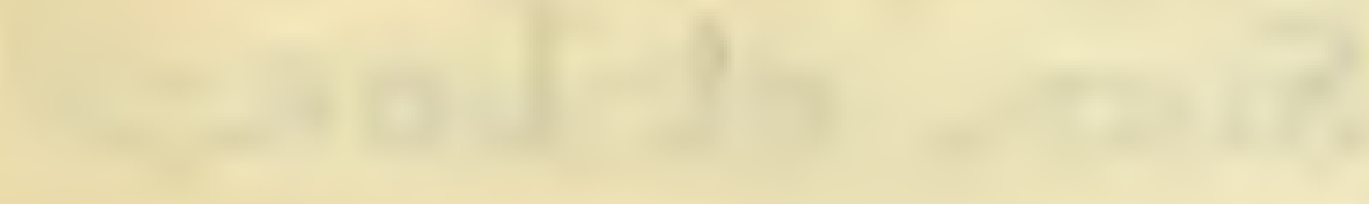

Drukarnia Państwowa w Poznania.

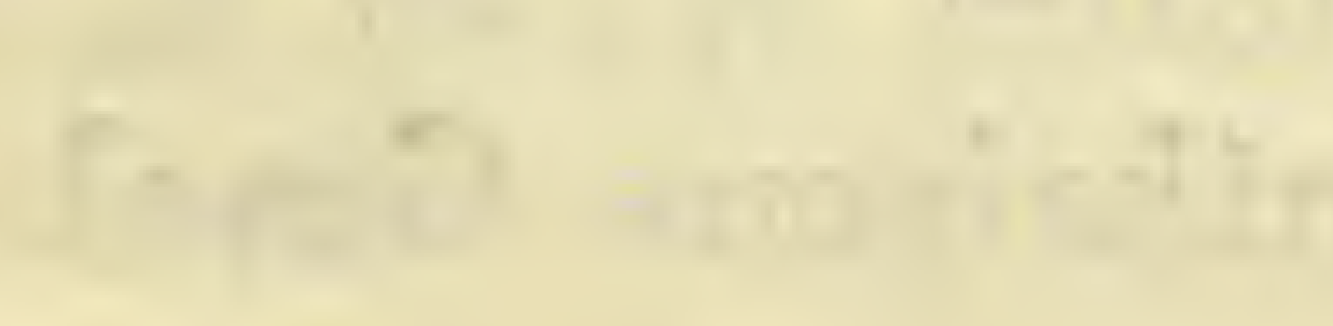

000000 $1712 \quad 29 / 30.500 .000>00$

$=$

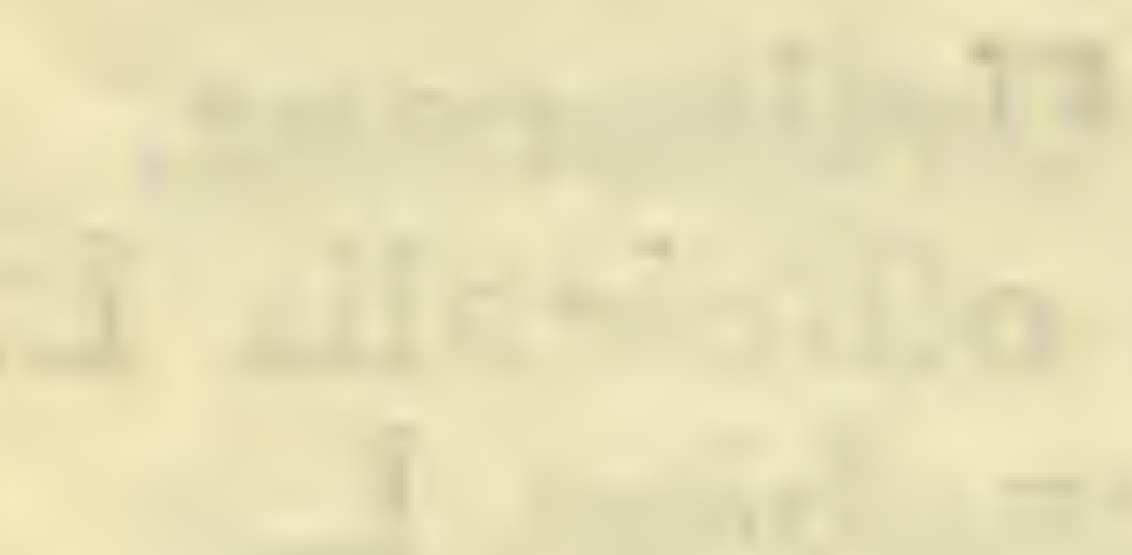

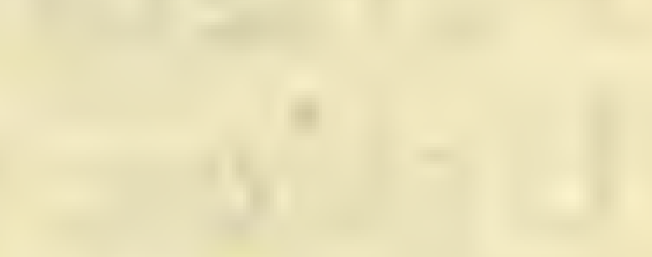

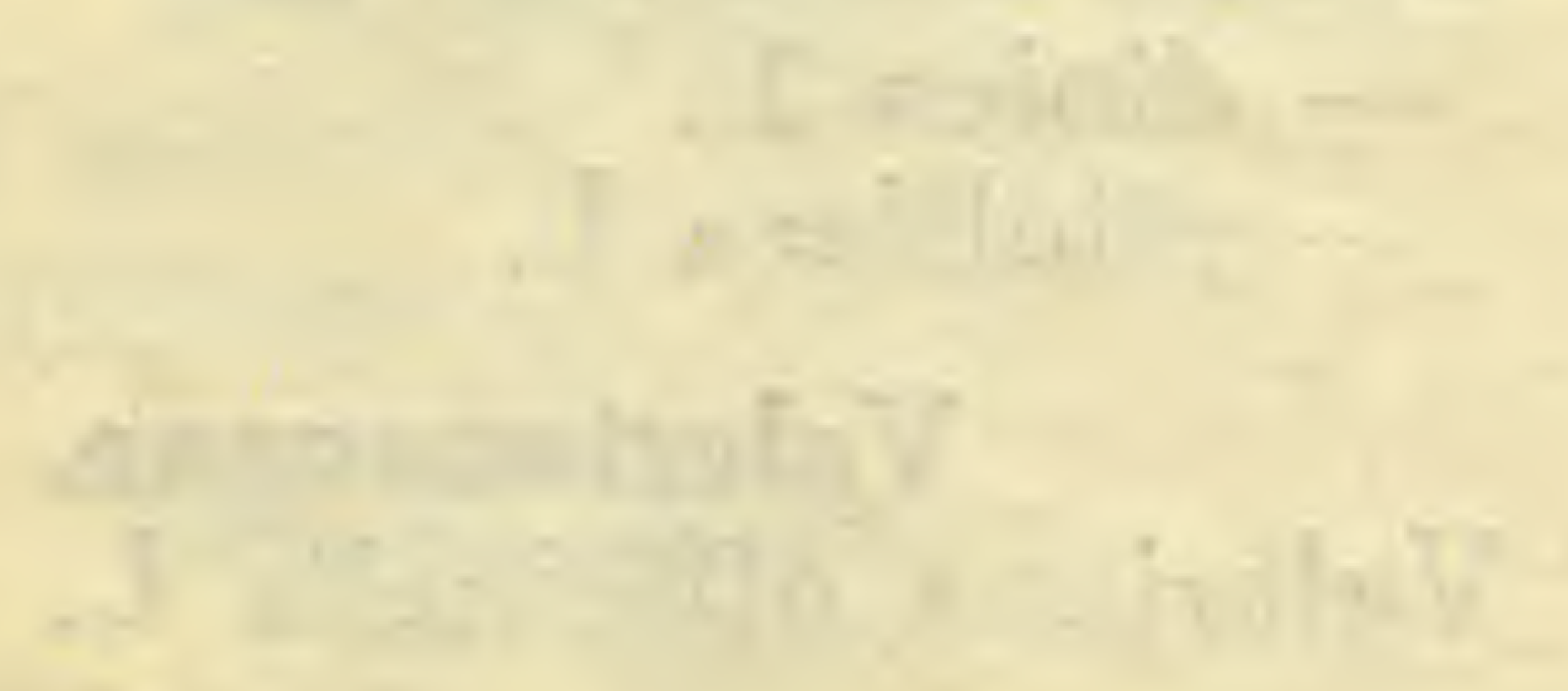

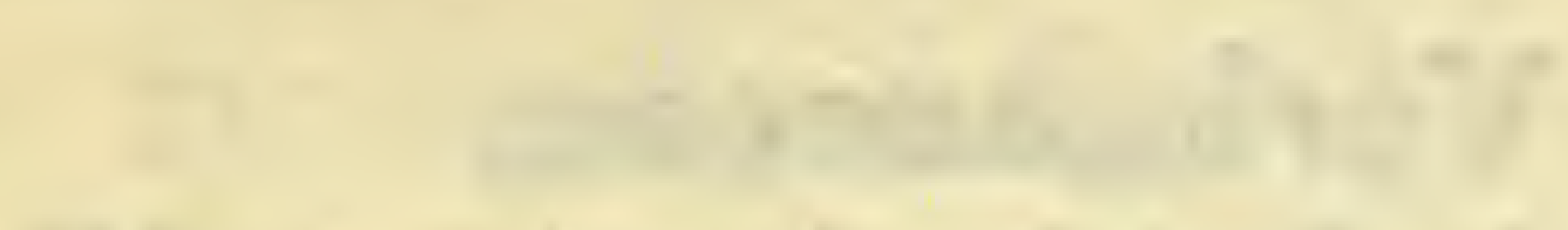

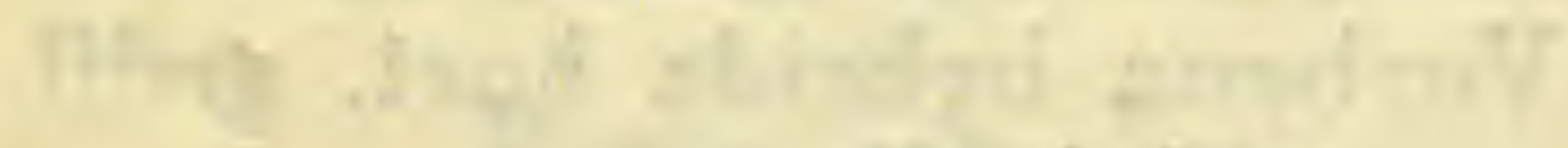

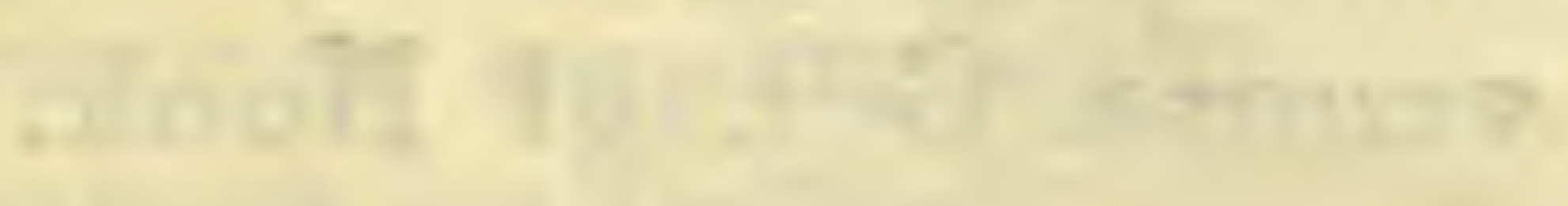

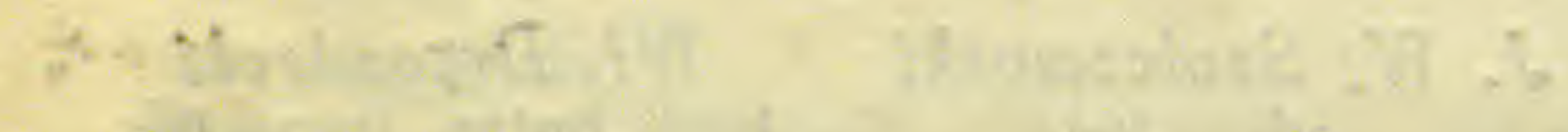

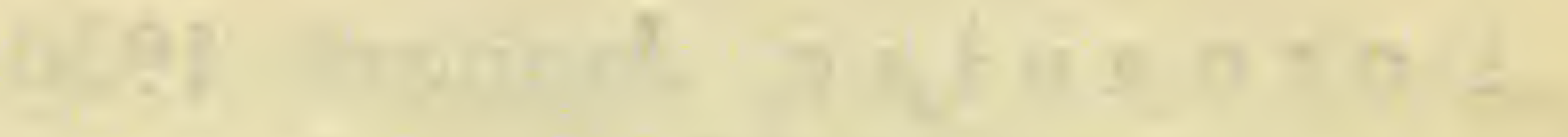

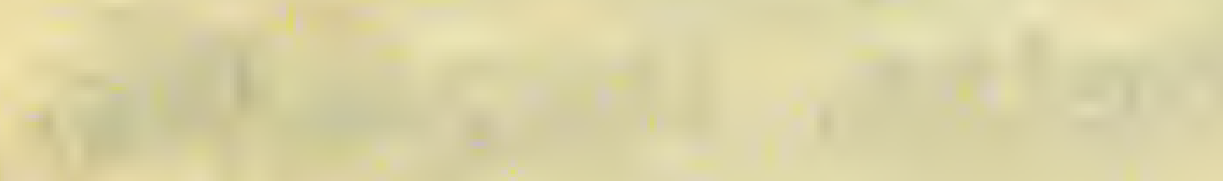

